Sādhanā Vol. 38, Part 6, December 2013, pp. 1287-1337. (C) Indian Academy of Sciences

\title{
Crystallization: Key thermodynamic, kinetic and hydrodynamic aspects
}

\author{
SREEPRIYA VEDANTAM* and VIVEK V RANADE \\ Industrial Flow Modeling Group (IFMG), Chemical Engineering and Process \\ Development Division, CSIR - National Chemical Laboratory, Pune 411 008, India \\ e-mail: sk.vedantam@ncl.res.in; vv.ranade@ncl.res.in
}

MS received 10 July 2012; revised 11 October 2012; accepted 17 October 2012

\begin{abstract}
Crystallization is extensively used in different industrial applications, including the production of a wide range of materials such as fertilizers, detergents, food and pharmaceutical products, as well as in the mineral processing industries and treatment of waste effluents. In spite of the wide-spread use of crystallization, a clear understanding of the thermodynamic, kinetic and hydrodynamic aspects of the design methodologies are not yet well established. More often than not crystallization is still considered an art especially in fine-chemicals, pharmaceuticals and life-sciences sector. It is essential to understand and relate key thermodynamic, kinetic and hydrodynamic aspects to crystallizer performance, not just in terms of yield but also in terms of product quality (characterized by particle size distribution, morphology, polymorphism and the amount of strain as well as the uptake of solvent or impurities in the crystal lattice). This paper attempts to do that by critically reviewing published experimental and modelling studies on establishing and enhancing state-of-the-art thermodynamic, kinetic and hydrodynamic aspects of crystallization. Efforts are made to discuss and raise points for emerging modelling tools needed for a flexible design and operation of crystallizers and crystallization processes that are needed to meet the ever increasing demand on precise product specifications. Focus is on bringing out the trends which can be used as perspectives for future studies in this field.
\end{abstract}

Keywords. Crystallization; supersaturation; nucleation; growth; polymorphism; CFD.

\section{Crystallization: Practice and key issues}

Industrial crystallization is gaining increasing attention in the chemicals, pharmaceuticals and fine chemicals industry. In these, as well as other branches, it is a crucial process step towards

*For correspondence 
particulate products with controlled properties. A major fraction of the fine and specialty chemicals, and pharmaceuticals manufactured today are produced and sold in a solid form. Increasing requirements on quality and consistency of such solid products have placed new demands on crystallization process steps.

There are two essential steps in crystallization process: nucleation and crystal growth. These steps essentially govern the Crystal Size Distribution (CSD). Mass transfer of a solute from super-saturated liquid solution to a pure solid crystalline, and mixing, can have a dramatic effect on product size distribution, purity and morphology. Supersaturation is defined as the excess dissolved solute with reference to the solubility curve. The overall crystallization performance therefore crucially depends on interactions of thermodynamic, kinetic and transport processes (which in turn depend on underlying hydrodynamics). The ability to control and to manipulate crystallizer performance therefore requires fundamental understanding of these aspects. In this work, we have attempted to provide a concise and critical review of available information on thermodynamic, kinetic and hydrodynamic aspects of crystallization.

Several reviews on crystallization including some classic overviews are available which provide comprehensive details on the crystallization process (see for example, Mullin 2001; Myerson 2002). The present review has been planned in order to bring forth the key issues involved in crystallization processes; the extent to which they have focused on reviewing the state of the art understanding of thermodynamic, kinetic and hydrodynamic aspects, and identifying the knowledge gaps therein that pose challenges to research as well as industrial requirements. The section is organized as follows: first, crystallization practice in industry is briefly reviewed and key issues are identified (section 1.2). The overall outline of the review is provided in section 1.3.

\subsection{Crystallization practice in process industries}

The first step in industrial application of crystallization is often to examine equilibrium data and select appropriate mode of crystallization. A typical solubility curve which determines the mode of crystallization to be employed, in order to crystallize a particular substance is shown in figure 1 (Jones 2002). If the curve is steep, i.e., the substance exhibits a strong temperature dependence of solubility (e.g., many salts and organic substances), then a cooling crystallization might be suitable. But if the metastable zone is wide (e.g., sucrose solutions), addition of seed crystal might be necessary. This may be desirable, particularly if a uniformly sized product is required. If on the other hand, the equilibrium line is relatively flat (e.g., for aqueous common salt solutions), then an evaporative process might become necessary. If the yield from either of the processes is low, then a second solvent can be added to reduce the effectiveness of the first and thus decrease the residual solution concentration (sometimes called 'drowning-out' or 'watering-out'). If the solute occurs as a consequence of chemical reaction or addition of a common ion, and is relatively insoluble, then precipitation or 'fast crystallization' occurs. Typically, the driving force for crystallization, supersaturation is generated by anti-solvent addition or cooling. In batch crystallization, a crystalline product with uniform size and shape is desirable, as the CSD determines the efficiency of various downstream processes. Control of supersaturation, by adjusting the cooling or addition of anti-solvent will determine the final crystal properties.

Many different types of crystallizers are used, depending upon the mode of crystallization. Figure 2 shows the different commercial crystallizers existing till date. For evaporative and cooling crystallization from solution, where the crystallizer is operated in continuous mode in 


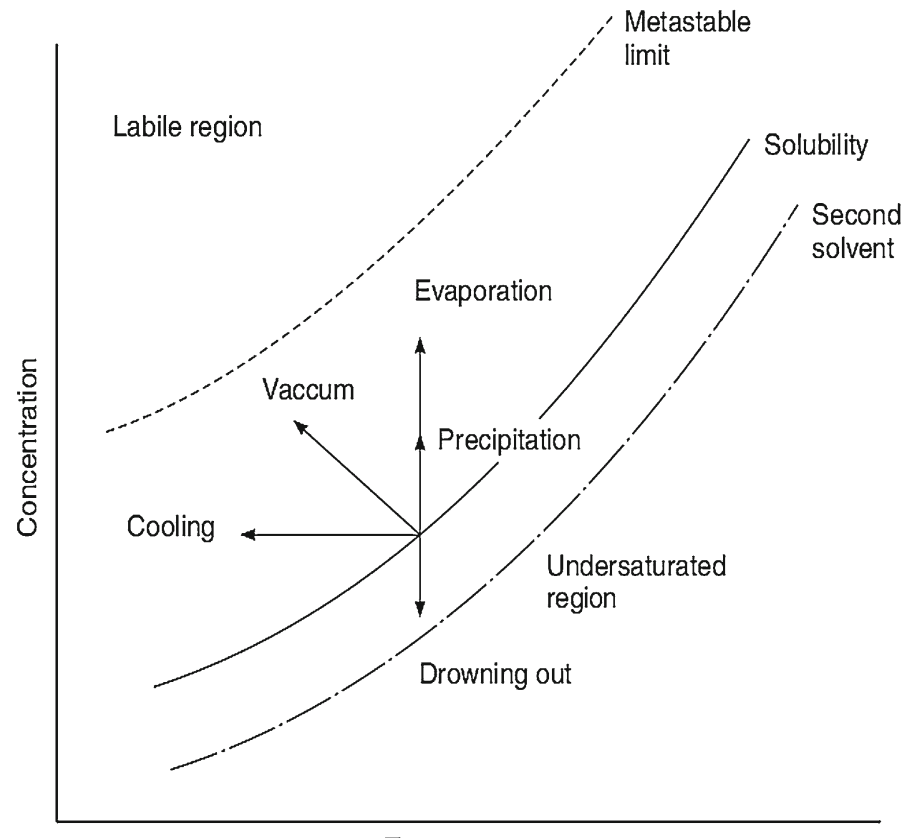

Temperature

Figure 1. Solubility-super solubility diagram (Jones 2002); Undersaturated-where crystals dissolve; Metastable-supersaturated region where crystals grow; Labile-region where solution spontaneously nucleates.

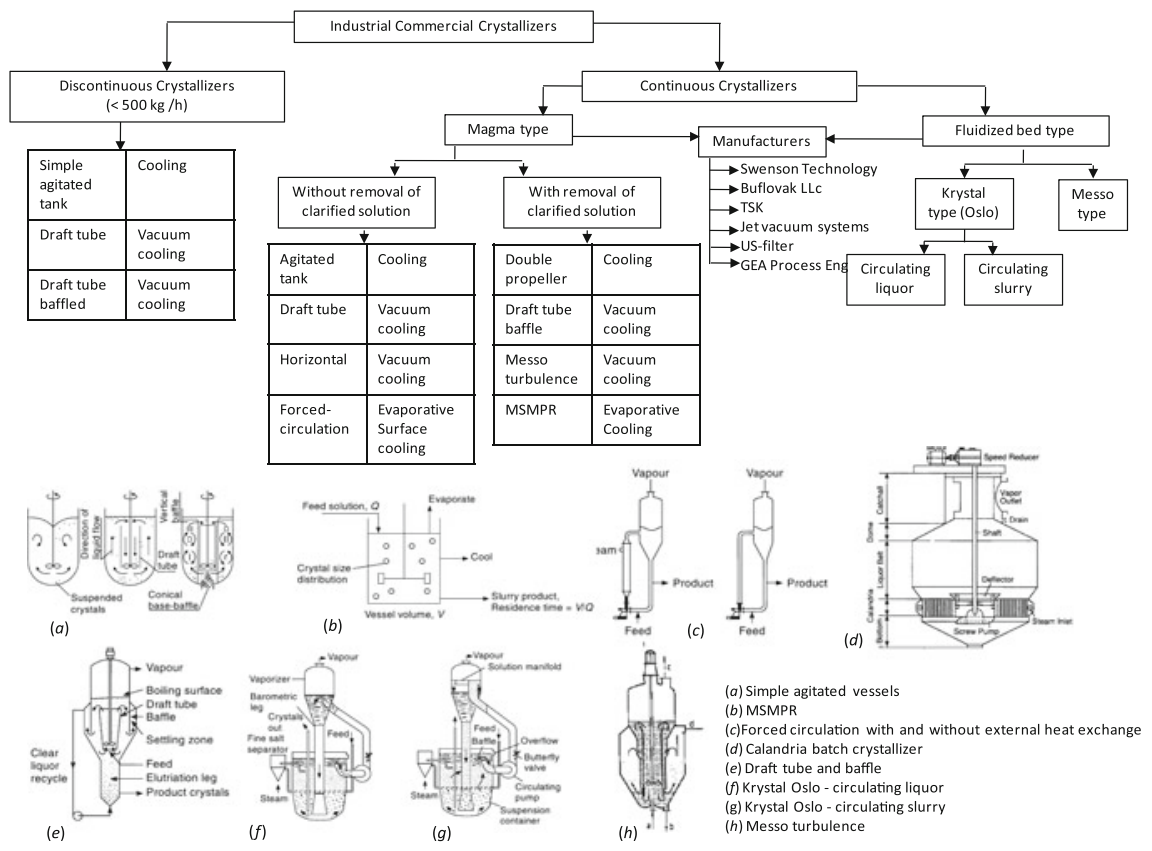

Figure 2. Details of the various types of industrial commercial crystallizers, including the established manufacturers of crystallizer units. 
general, three types of industrial crystallizers dominate: Forced Circulation (FC), Draft-TubeBaffled (DTB) and Fluidized Bed. In an FC crystallizer that is particularly designed for largescale production by evaporation, the externally heated suspension reaches the boiling zone through either a tangential or an axial inlet. The main design problem is that with these inlet types, the full cross sectional area of the crystallizer is not used for evaporation. This leads to thermal short-circuiting or liquid entrainment to the condenser and puts constraints on the circulation flow. In case of a tangential inlet also, vortex formation limits this flow rate. Later, new radial inlet nozzle for FC crystallizers was introduced (Dimonte et al 2000).

The design of fluidized bed crystallizers is meant to produce large and uniformly sized particles. But specific production rate was found to be rather low in comparison to FC or DTB crystallizers. One reason for this, as given in the literature, is the restriction of the circulation velocity by the requirement that a fluidized bed has to be maintained. Another reason is that the supersaturation of the clear liquid in the down-comer has to be kept low to avoid scaling by heterogeneous primary nucleation on the (down-comer) walls (Kramer \& Jansens 2003). Because of the high circulation velocities, this crystallizer can also be used as a cooling crystallizer without too much trouble in the heat exchangers. In the past two decades, a good number of industrial applications of melt crystallization came into practice. A lot of research has been done and the fundamental understanding of melt crystallization has increased considerably (Jansens \& Matsuoka 2000). In late 1990s and early 2000s, some application based studies were focused on crystal-liquor separation, and thus to improve the energy-efficiency (Jans et al 1996; Diepen 1998), focusing on flash-cooling melt crystallizers. Early 2000s brought into practice, fluidized steel beads and hydraulic wash columns to remove encrustation from the cooled surface.

The importance of precipitation as a technique for recovery of products from process streams and the formation of particulate products has increased considerably over the past years in the wake of the fast growing fine-chemicals sector in general and the pharmaceutical industry in particular. In this branch of industry, the crystallizers are usually simple stirred vessels, which are operated batch-wise for a variety of products. There is a growing awareness of the fact that local hydrodynamic conditions have a major impact on the rates of nucleation and agglomeration and consequently on the product quality, as well as on the type of polymorph that is formed. Primary nucleation occurs mainly at the inlet streams, and consequently the interest in feed-dilution systems, premixing devices has risen. If the appropriate particles are obtained by applying a particular type of inlet device in combination with a residence vessel for further outgrowth and agglomeration, the problems inherent to scaling up can be avoided by the simple use of several devices connected to one larger residence vessel. Because precipitation is only applied for slightly soluble compounds, clear liquor advance is frequently used to increase the solid content in the vessel. The additional difficulties of separation of small agglomerates were of course overcome later, which are not covered within this manuscript.

Applications were also identified in the field of water treatment, as well as in the pharmaceutical industry. Supercritical precipitation processes, where condensed gases or near-critical fluids are used as solvents or anti-solvents, have received broad attention from the academic research as well as from the industry (Jung \& Perrut 2001). Promising applications also include production of medical drugs and the micronization of fats, waxes and polymers. Research in this field of industrial crystallization has been active in the past decade, but process development and equipment design is constrained by the limited reliable data and modelling frameworks on the combination of thermodynamic, kinetic and the hydrodynamic aspects involved in these applications. 


\subsection{Key issues}

The essentials of crystallization are based on nucleation and crystal growth and the factors influencing them. Together, these processes govern the CSD by determining the number and size of crystals present in the crystallizer at the end of operation. Mixing affects the product property and quality, including the CSD, purity, morphology and polymorphic form. These parameters also influence the choice of further downstream processing. Nucleation is the formation of a new crystal by either primary or secondary mechanisms. Primary nucleation, occurring at high level of supersaturation, is a formation of nuclei in a crystal-free solution. As crystal formation progresses, the new nuclei are often formed by a combination of primary nucleation at the feed point and secondary nucleation in the bulk of the vessel. The radius of the nuclei must be large enough to overcome the free-energy activation barrier, such that growth can occur. Smaller clusters will dissolve. Usually, a large supersaturation driving force is required to initiate nucleation, and in many cases, results in the formation of a large number of nuclei. The precise mechanism by which these clusters form is either not very well understood or scantly available in the literature. Primary nucleation along with secondary effects such as agglomeration, breakage and ripening are usually the main mechanisms dictating the CSD and morphology of the crystal formed.

The need to control polymorph formation has been an important issue in chemical and pharmaceutical industry over a century. Although identical in chemical composition, polymorphs differ in bioavailability, solubility, dissolution rate, chemical stability, physical stability, melting point, colour, filterability, density, flow behaviour and other properties. In particular, variation in solubility between different polymorphs is important for pharmaceuticals, as it can affect drug efficacy, bioavailability and safety.

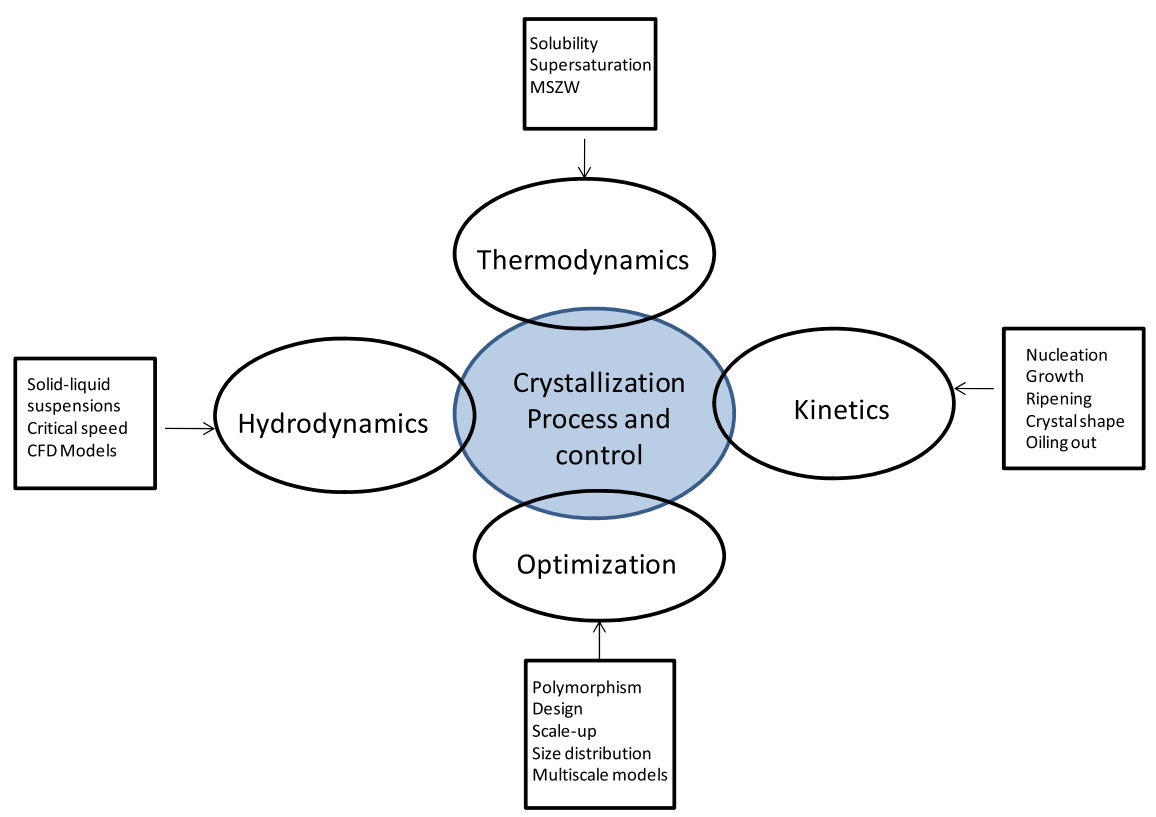

Figure 3. Interplay of various modelling aspects in crystallization process, and various corner stones in each modelling section. 
Today, crystallization modelling tools are limited by the availability of methods in reliably predicting solubility and supersaturation for chemical systems of industrial importance. There is also limited capability for describing nucleation and crystal growth, and mixing and design scale-up for industrial crystallizers. Tools are needed to model and optimize crystallizer process performance with respect to product quality, throughput and efficiency. The interplay of various modelling aspects involved in crystallization process is given in figure 3 . Table 1 presents the crystallization modelling aspects addressed in the past, along with the major research groups that have carried out the work. While, table 2 presents the various experimental techniques used for the understanding of crystallization process, and the research groups with their affiliation, that have carried out research using those techniques.

Table 1. Various modelling aspects of crystallization modelling and major research groups, across the globe.

\begin{tabular}{|c|c|c|c|}
\hline \multicolumn{4}{|c|}{ Crystallization modelling } \\
\hline Modelling type & Platform & Group & Affiliation \\
\hline \multirow{3}{*}{$\begin{array}{l}\text { Temperature and } \\
\text { concentration control } \\
\text { of batch crystallization }\end{array}$} & \multirow{4}{*}{$\begin{array}{l}\text { MOSCED, } \\
\text { UNIFAC }\end{array}$} & Braatz R D & \multirow{3}{*}{$\begin{array}{l}\text { University of Illinois } \\
\text { at Urbana-Champaign, USA } \\
\text { TU Dortmund, Germany }\end{array}$} \\
\hline & & Sadowski G & \\
\hline & & Rasmuson A C & \\
\hline $\begin{array}{l}\text { Thermodynamic } \\
\text { modelling }\end{array}$ & & & $\begin{array}{l}\text { Royal Institute of } \\
\text { Technology, Sweden }\end{array}$ \\
\hline $\begin{array}{l}\text { Process control and } \\
\text { quality monitoring }\end{array}$ & ICAS-PAT & $\begin{array}{l}\text { Gernaey K V } \\
\text { Gani R }\end{array}$ & $\begin{array}{l}\text { Technical University } \\
\text { of Denmark }\end{array}$ \\
\hline \multirow[t]{3}{*}{ CFD } & \multirow[t]{3}{*}{ Fluent, CFX } & Marchisio D L & University of Turino, GSK \\
\hline & & Ricard F & \multirow{2}{*}{$\begin{array}{l}\text { Aristotle University } \\
\text { of Thessaloniki, Greece }\end{array}$} \\
\hline & & Kipparissides C & \\
\hline \multirow[t]{3}{*}{ Molecular modelling } & & Jansens P J & TU Delft, The Netherlands \\
\hline & & Kramer H J M & \multirow[t]{2}{*}{ University of Michigan, USA } \\
\hline & & Hornedo N R & \\
\hline \multirow{4}{*}{$\begin{array}{l}\text { Population balance } \\
\text { model }\end{array}$} & \multirow{4}{*}{$\begin{array}{l}\text { PARSIVAL } \\
\text { (Particle size } \\
\text { evaluation) }\end{array}$} & Nieken U & Computing in Technology \\
\hline & & Hounslow M J & GmbH, Germany \\
\hline & & Rawlings J & University of Sheffield, UK \\
\hline & & Witkowski W S & University of Texas at Austin, USA \\
\hline \multirow[t]{7}{*}{ Kinetics modelling } & \multirow{3}{*}{$\begin{array}{l}\text { In-house } \\
\text { Monte-Carlo } \\
\text { simulation }\end{array}$} & \multirow[t]{3}{*}{ Briedis Zauner R } & Michigan State University, USA \\
\hline & & & University college London, UK \\
\hline & & & Indian Institute of Science, India \\
\hline & \multirow{4}{*}{$\begin{array}{l}\text { Parameter } \\
\text { estimation of } \\
\text { kinetic parameters }\end{array}$} & Madras G & EPFL, Switzerland \\
\hline & & Ring T A & TU Delft, The Netherlands \\
\hline & & Kramer H J M & University of California \\
\hline & & Doherty M F & at Santa Barbara, USA \\
\hline \multirow[t]{2}{*}{ Micromixing model } & \multirow{2}{*}{$\begin{array}{l}\text { Inter-exhange with } \\
\text { mean coupled } \\
\text { with DQMOM }\end{array}$} & Marchisio D L & Politecnico di Torino, Italy \\
\hline & & Baldyga $\mathrm{J}$ & $\begin{array}{l}\text { Warsaw University } \\
\text { of Technology, Poland }\end{array}$ \\
\hline \multirow{3}{*}{$\begin{array}{l}\text { Multiscale, multizonal } \\
\text { models }\end{array}$} & \multirow[t]{3}{*}{ CAPE-OPEN } & Pantelides C C & Imperial college London, UK \\
\hline & & Ramagnoli J A & University of Sydney, Australia \\
\hline & & Kindermann S & University of Linz, Austria \\
\hline
\end{tabular}


Key thermodynamic, kinetic and hydrodynamic aspects

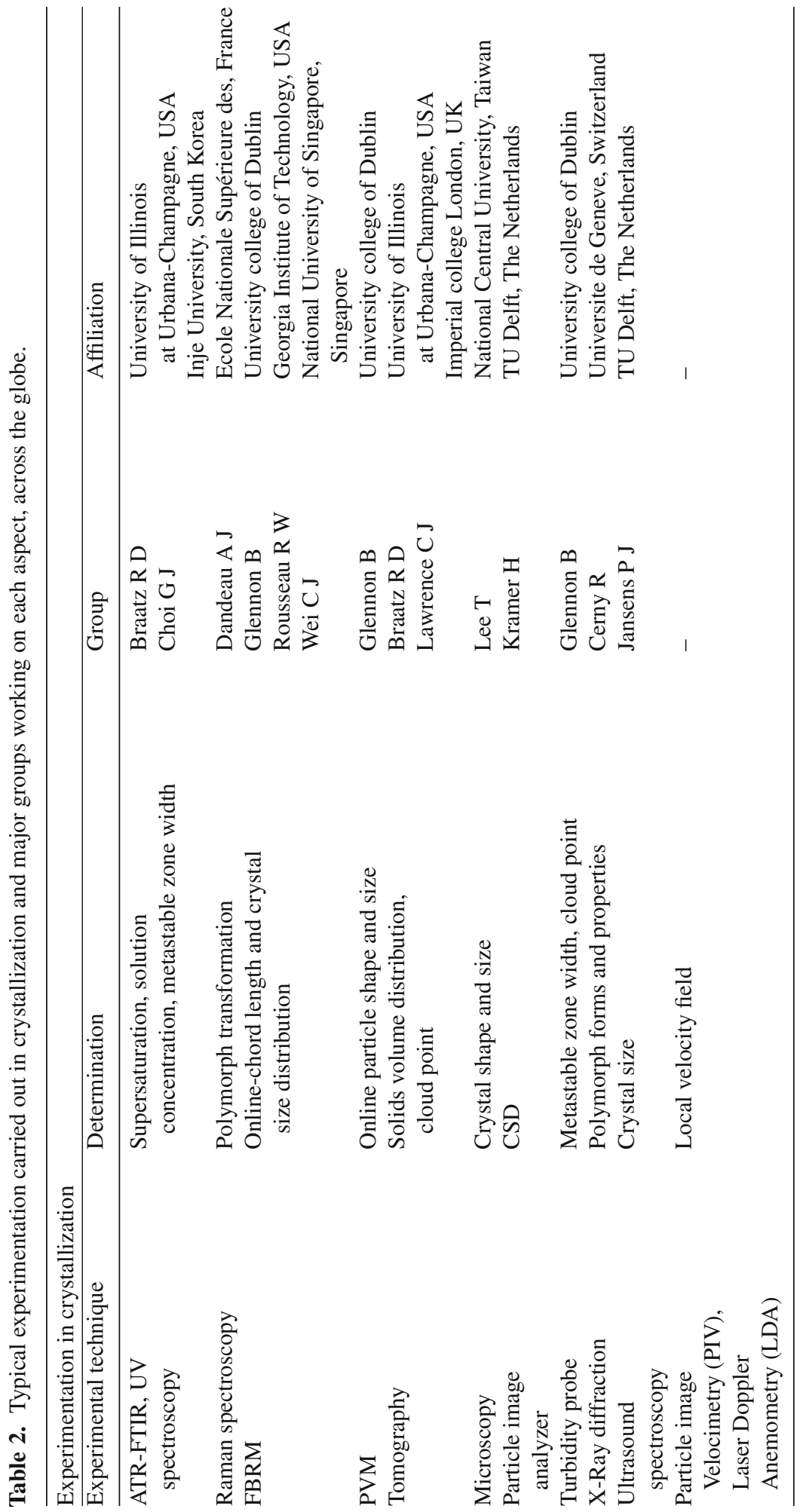




\subsection{Organization of this paper}

In order to address these limitations, and further understand the processes occurring in depth, it is thought desirable to carry out a detailed and critical review of the modelling and experimental studies published so far in literature, which includes CFD modelling of liquid-solid flows, Population Balance Modelling (PBM) for the determination of CSD, and reaction engineering models to obtain the desired thermodynamic conditions and comprehensive overview of the experimental methods used to determine solubility, supersaturation, metastable zone, polymorphism and CSD. Finally, a framework for entire modelling process applied to crystallization is attempted and is presented. An integration of these modelling methods would lead to a platform for an in-depth process understanding.

In subsequent sections, the key aspects involved in understanding of crystallization process are covered in detail. Section 2 covers the thermodynamic aspects focusing on solubility, metastable zone width and supersaturation studies. Studies in literature are analyzed in terms of both experimental and modelling studies. In sections 3 and 4, the kinetic and hydrodynamic aspects are covered in detail. After discussing thermodynamic, kinetic and hydrodynamic aspects, crystallization control, design and scale-up aspects are discussed in the subsequent section. Key conclusions and suggestions for further work are offered at the end.

\section{Thermodynamic aspects}

Crystallization process mainly bases itself on the thermodynamic behaviour of the system. These characteristics include solubility, supersaturation and the metastable zone; which play a role on subsequent kinetics. An understanding of solubility is the starting point for crystallization design, forming a key aspect to decide the throughput and yield. Also, it plays a crucial role in solvent selection system as well as the mode of crystallization. The next step being, an understanding of metastable characteristics, refer to solutions that are supersaturated, yet are not ready to nucleate spontaneously. These aspects are covered in detail in the subsequent sections.

\subsection{Equilibrium solubility}

As explained earlier in figure 1, the different means of crystallization can be decided based on the solubility diagram. Solubility is a dynamic equilibrium whereby the rate of dissolution is balanced by the rate of crystallization. It is the maximum concentration that can exist at equilibrium at a given set of conditions and often increases with an increase in solution temperature. Hence, it can be said that the temperature coefficient of solubility is an important factor which could determine the crystal yield; since if the coefficient is positive and increasing, it would imply that an increase in temperature leads to more solute solubility and thus more solution concentration. For a substance to dissolve in a liquid, it must be capable of disrupting the solvent structure and permit the bonding of solvent molecules to the solute component ions. The forces binding the ions, atoms or molecules in the lattice oppose the tendency of a crystalline solid to enter solution. The solubility of a solid is thus determined by the resultant of these opposing effects.

Primary limitation in controlling crystallization process $(\mathrm{CP})$ and systemic modelling of the process is the accurate in situ measurement of solute concentrations in the slurries. High accuracy is needed as the nucleation and growth kinetics which are basic for $\mathrm{CP}$, are strongly dependent on supersaturation, which is the difference between actual solute concentration and saturated solute concentration. The details on supersaturation and its role in crystallization process are discussed 
in subsequent sub-section 2.2. As for the measurement of solubility, various techniques were in use which were triggered off in early 1970s. Yet, there is no single measurement technique that is applicable to all systems in general.

Refractive index measurement was one of the techniques used, however, when there existed large difference in presence of solute, yet, in presence of ambient light and air bubbles, this method did not find its use (Helt \& Larson 1977; Mullin \& Leci 1972). Another approach was to sample out the crystal slurry, filter the crystals and determine the density of the liquid phase. The use of external sampling loop led to the operational difficulty of clogging as well as temperature sensitivity (Miller \& Rawlings 1994; Redman \& Rohani 1994). This was later identified to cause inaccuracies in the solute concentration measurements, especially in smaller metastable zone width crystals wherein small temperature differences led to nucleation (Togkalidou et al 2001). In processes involving crystallization of electrolytes, conductivity measurements were also carried out to estimate solute concentration (David et al 1991; Garcia et al 1999). This method also offered limitation in calibration of probe performance in longer term usage. Calorimetry was another indirect measurement approach in which temperature and flow rate measurements are combined with the overall energy balance of the crystallizer. These estimates drifted away as the solute concentration varied, while the crystallization process $(\mathrm{CP})$ progressed (Févotte \& Klein 1994, 1996).

Togkalidou et al (2001) demonstrated the advantage of spectroscopic techniques for the determination of solution concentrations with its applicability to multicomponent systems. It was demonstrated in their work that FTIR-ATR spectroscopy with the proper application of experimental design and robust chemometrics can provide highly accurate measurements of solutions in slurries of KDP crystals. Using a wide range of frequencies applicable within IR range, they could attain confidence interval of $95 \%$, determining solution concentration upto $\pm 12 \%$ by weight. McKeown et al (2011), in a comprehensive overview on crystallization design and scaleup, presented the common crystallization modes and the influence of solubility behaviour on the selection of modes.

The major experimental difficulties in any of the methods mentioned above are the achievement of equilibrium in the solution. Prolonged agitated contact is required between solid solute and solution at a constant temperature, usually for several hours or days (according to system temperature and viscosities). Approaching equilibrium from both the under-saturated and the over-saturated states can however, check the accuracy of the solubility determination.

In general, the experimental determination of solubility of pharmaceuticals in various organic solvents requires a large amount of the solute and is usually a time-consuming procedure. In many pharmaceutical applications there are only a few milligrams of a solid available; therefore, solubility prediction using thermodynamic models is the preferred choice. The thermodynamic properties of liquid-vapour, liquid-liquid, and solid-liquid chemical systems found by using various methods (for example group-contribution methods or regular-solution theory) are reported in the literature (Hojjati \& Rohani 2006). However, the solubility prediction of pharmaceutical solids in various solvents has received less attention. Solubility prediction needs an estimation of the ideal solubility and activity coefficient of the solute in a particular solvent. The solubility prediction is a fundamental aspect of any crystallization model, because its prediction is required for the calculation of supersaturation, which in turn, is used for determining nucleation, growth, and other crystallization phenomena.

A good solubility model accurately predicts how the equilibrium concentration of the solute changes over the course of the crystallization batch. This accurate solubility prediction is required for a crystallization model to in turn become accurate in predicting crystal product properties like size (Widenski et al 2010). Solubility models can be based on either empirical or 
thermodynamic foundations. An empirical solubility model is an equation fitted to experimental solubility data, and typically has no underlying physical meaning, while on the other hand, a thermodynamic solubility model both fits the data and has physical meaning. Empirical solubility models have been extensively used in crystallization modelling, as reported in the literature (Nowee et al 2008; Zhou et al 2006).

Common types of thermodynamic models include those based on excess Gibbs energy such as Wilson, NRTL, or UNIQUAC. In addition, predictive thermodynamic models can also be used such as MOSCED, NRTL-SAC, or UNIFAC. The advantage of these predictive models is that no new experimental data is needed to calculate activity coefficients. The first predictive thermodynamic model considered is the MOSCED (Modified Separation of Cohesive Energy Density) model. The MOSCED model is a thermodynamic model used to calculate infinite dilution activity coefficients. The advantage of the MOSCED model is that no experimental data is needed to calculate the infinite dilution activity coefficients. The MOSCED model further calculates temperature dependent infinite dilution activity coefficients, such that a temperature-dependent activity coefficient model is not required. The MOSCED model was originally developed for binary liquid solutions, but was later extended to liquid-solid systems by Draucker et al (2007).

The choice of the best thermodynamic model for liquid-solid equilibria is not an easy task and most of the available commercial software is not originally built for this. The available thermodynamic models are based on various approaches. UNIFAC and its modifications are group contribution methods. They only require binary interaction parameters between the functional groups. When these parameters are not available, these models cannot be used. COSMO-SAC is based on quantum mechanics and requires profiles of the charge density around the studied molecules. The challenge is the determination of the best profiles, which take into account the right conformation of the molecules in the solution. NRTL-SAC is based on polymer NRTL and to be used, four parameters representing the behaviour of the molecules have to be calculated from experimental data regression. Once these parameters have been calculated, for one molecule, they can be used for any other solubility prediction in any solvent. There is no need to regress each time, like in most of the semi-predictive models like UNIQUAC or Wilson. However the main challenge in this field is to improve the accuracy of the models in order to be able to predict the solubility of molecules in complex solvents, the solubilities of solutes and also to be able to describe oiling out behaviour (formation of an additional phase, while the crystallization starts to occur: this is covered in detail in section 3.5), and to model phase equilibrium.

2.1a Effect/choice of solvent: Along with solubility characteristics and supersaturation, there have been studies reporting the use of solvents or solvent mixtures in order to control the polymorph formation (Blagden et al 2005; Davey et al 2002). The type and choice of solvent plays a major role in polymorphic selectivity and crystal morphology of the product (Rohani et al 2005), mainly due to solvent-solute interactions at the molecular level. This was previously shown in early 1970s that the solvent can facilitate favourable interactions with solute clusters or nuclei on specific growing faces, which cause reduction in interfacial tension, thus improving the pace of crystal growth. Rohani et al, also specified that the solubility of crystallizing solute should be in the range of $5-200 \mathrm{mg} / \mathrm{mL}$ at room temperature, in order to make the solvent conducive for crystallization process. The choice of the solvent though could be governed by solvation power, slope of solubility curve versus temperature, boiling point, viscosity, and ability to form hydrogen bonding; along with the safety aspects.

The effect of solvents on polymorphism has not been very clearly depicted in the literature, though this was thought beneficial for desirable control of polymorphism. Mirmehrabi et al 
(2006) developed correlations to determine the ability of solute and solvent molecules to form intra- and intermolecular hydrogen bonds.

\subsection{Supersaturation}

The fundamental, thermodynamic, driving force for crystallization, or precipitation, is given by the change in chemical potential between standing and equilibrium states. Chemical potential is a quantity that is not so easy to measure however, and hence in the literature over the years, the driving force is more conveniently expressed in terms of solution concentration as per the following approximation:

$$
\Delta \mu=\ln \left(\frac{c}{c^{*}}\right) \cong \frac{c}{c^{*}}-1=\frac{\Delta c}{c^{*}}=S-1=\sigma,
$$

where $\Delta \mu$ is the change in chemical potential, $c$ is the standing concentration and $c^{*}$ the equilibrium saturation concentration, $S$ is the supersaturation ratio and $\sigma$ is the relative or absolute supersaturation. It is worth noting that although commonly used, the above equation is strictly valid only for $c \approx c^{*}$, but many precipitations employ $c \gg c^{*}$. Supersaturation can be thought of as the concentration of solute in excess of solubility. For practical use however, supersaturation is generally expressed in terms of concentration

$$
\Delta c=c-c^{*},
$$

where $c=$ concentration of solution, $c^{*}=$ saturation concentration and $\Delta c$ is sometimes called the 'concentration driving force'. Supersaturation is defined as the difference between the solution concentration and the equilibrium concentration (absolute supersaturation), or as the ratio of the two (relative supersaturation).

For ionic systems however, the definition of the appropriate driving force becomes more complex since the ionic concentrations are not necessarily in stoichiometric ratio and the solubility product generally applies. Sparingly soluble substances can easily exhibit high levels of relative supersaturation. Hence, the crystallization process can be very fast and the precipitation difficult to control, especially on the large scale (see Sohnel \& Garside 1992).

Crystal growth rate (typically termed $\mathrm{G}$ and expressed in terms of micrometers per unit time) is a function of supersaturations higher the supersaturation, higher is the growth rate. Additionally, nucleation or formation of fine crystals (typically termed B, in terms of birth of new crystals per unit time) is also driven by the supersaturation. Therefore, controlling the supersaturation level over time can aid control of the levels of growth and nucleation achieved, thereby presenting the opportunity to control the size of the crystals produced. Certainly for a production process, having high levels of both growth and nucleation will result in a very broad CSD, which is undesirable from a downstream handling perspective.

Control of supersaturation either by cooling or anti-solvent addition basically determines the final crystal properties. Two main approaches exist to determine the most optimal profiles for supersaturation. In model based control, a robust model to completely describe the crystallization process is needed. Based on this model, theoretical optimal cooling and anti-solvent addition profiles can be determined. The direct feedback approach involves maintaining a constant predefined supersaturation by measuring concentration throughout the crystallization process via in situ sensors. The supersaturation is kept below the MSZL, defined as supersaturation at which nucleation will occur. Both approaches have been applied extensively to cooling and anti-solvent crystallization (Knox et al 2009; Hu et al 2005; Feng \& Berglund 2002; Foddi et al 2007; Nowee et al 2008). Various works reported in the literature on the effect of supersaturation rate on other 
aspects of crystallization such as the metastable zone width and nucleation, are covered in the subsequent sections.

2.2a Seeding: Seeding a solution with a crystal of the product is a well-established method to induce crystallization (Dunitz 2003). The addition of small 'seed' particles to a supersaturated solution can greatly increase the rate at which crystals nucleate. This method was also used to choose the formation of a specific type of polymorph provided the desired product seed-crystal was available. In order to use the seeding technique efficiently, it is essential to understand the width of the metastable zone and the range of conditions over which the solution can be supersaturated.

Key variables in seeding include seed loading (i.e., wt $\%$ of seed), seed size, and method of seed addition. Typically, the smaller the seeds, the higher the surface area per unit mass, and hence more is the opportunity for a growth-dominated process. Large crystals, relative to smaller ones, will provide less surface for growth and may result in secondary nucleation being the dominant crystallization mechanism during the process (Barrett et al 2005). It is difficult to generalize these areas specifically, but typically the smaller the seed size and higher the seed loading, the easier it is to "control" a crystallization process with the minimum of process upsets throughout all process development and production.

Kubota et al (2001) showed that in a seeded cooling crystallization, a unimodal CSD can be ensured if sufficient amounts of seeds are used, irrespective of the cooling method/conditions used. Llinas \& Goodman (2008) presented a detailed overview on the recent advances in seeding and other additives for use in polymorph control. They considered the effects of modifying a solvent, physical confinement of growing crystals and methods of obtaining supersaturated solutions. They also presented details on the use of radiation for stimulating crystallization. It was also shown in the literature that the initial concentration of solute and temperature while seeding, impacts the crystal yield (Al-Zoubi \& Malamataris 2003). Literature offers suggestions on the ways of addition of seed material. Seeds can be added dry or in slurry form. Adding the seed in slurry form (either in anti-solvent or mother liquor) may help the seeds disperse to their primary crystal size. Adding dry seeds may induce unwanted solvent entrapment and agglomeration, as the dry seeds may have aggregated during storage/preparation. Tung et al (2009) clearly summarized the various techniques that can be used as seeding methods.

However, the microscopic mechanism of seeding by a 'foreign' substance is not covered in the literature. In one of the very few studies reported, Cacciuto et al (2004) reported numerical simulations of colloidal crystallization seeded by foreign objects. They performed Monte Carlo simulations to study how smooth spherical seeds of various sizes affect crystallization in a suspension of hard colloidal particles, thus computing the free energy barrier needed for nucleation.

It is shown in the literature that to be effective crystallization promoters, the seed particles need to exceed a well-defined minimum size, beyond which these trigger the nucleation, thus detaching the newly formed nuclei from the seed. Though Cacciuto et al, state that larger seed particles remain covered by the crystallites that they bring about, this phenomenon has not yet been proven experimentally. However, it may have important influence on controlling the CSD.

\subsection{Meta stable zone width (MSZW)}

The MSZW is defined as the difference between the solute concentration and the saturation concentration $\left(C_{\text {Sat }}\right)$ or that between solution temperature and the temperature at which spontaneous 
nucleation can occur in a short time ( $\left.T_{\text {Sat }}\right)$ (Nyvlt et al 1985). The classic works of Kashchiev and co-workers in early 1990s defined the metastability limit as the critical supersaturation below which the induction time exceeds some threshold time: $t_{\text {induction }}\left(\Delta \mu_{c}\right)=t_{\text {threshold }}$. Determining the MSZW is important to avoid or minimize spontaneous nucleation and obtain a narrow CSD. It can be characterized by several methods, but most commonly as a clouding of the solution or "nuclei shower", which corresponds to particles reaching a detectable size. Defining the MSZW from an experiment with varying supersaturation raises the important possibility that increasing the supersaturation at different rates or that using different schedules for the supersaturation as a function of time might lead to different values for the MSZW. Experiments do find that MSZWs defined this way depend on the rate at which supersaturation increases. The MSZW can also be characterized using Focused Beam Reflectance Measurement (FBRM) and ATR-FTIR (Barrett \& Glennon 2002; Fujiwara et al 2001). Two standard approaches are used to determine MSZW. In the polythermal method, the solution is cooled at a constant rate until nucleation is detected. In this case, the MSZW is described using $C_{\text {Sat }}$ or $T_{\text {Sat }}$. For the isothermal method, a constant supersaturation is maintained until visible crystals are formed. The time between the generation of supersaturation and the formation of visible nuclei is defined as the induction time (Nyvlt et al 1985).

Despite the significance offered to MSZW in crystallization studies, there is no generic model developed for the prediction of this zone. It is dependent on a variety of factors such as presence of seeds or foreign particles, cooling rate, agitation rate, temperature, thermal history of solution, and reactor geometry. The MSZW is also greatly influenced by the type of dominating nucleation mechanism: primary, secondary, surface, or attrition (Kwang-Joo \& Mersmann 2001). Modelling studies have been reported in recent decade by Yang et al (2006). The MSZW is often modelled using power law equations for growth and nucleation. The model of Mersmann \& Bartosch (1998) assumes that the induction time, and hence maximum supersaturation, is a function of the integral of previous supersaturation. In that model the integral of supersaturation is equal to some constant value at the maximum supersaturation. Yang et al (2006) produced a similar model in which they considered the MSZW a function of the sum of previous events. They showed that their model could predict the MSZW when the cooling rate was non-linear, a case where traditional models failed. Later Nagy et al (2008) adapted the model of Mersmann to unseeded crystallization, taking into account the nucleation event.

\section{Kinetic aspects}

The majority of control approaches applied in crystallization affect the CSD indirectly by implementing a temperature or anti-solvent profile in time to follow a given supersaturation profile in the phase diagram. These profiles are obtained either using simple trial-and-error experimentation or more complex model-based or direct-design approaches. However, it is recognized that the variability in the final CSD is mainly caused by the significant uncertainties in the nucleation rate, and the above-mentioned approaches identify operating protocols, which provide an acceptable compromise between crystal growth and nucleation.

The study of polymer crystallization has also received considerable attention in the last three decades. Polymer crystallization controls the macroscopic structure of the material, and thereby determines the properties of final polymer products (Chen \& Higg 1998). The morphology of polymer crystals is different from that of crystals consisting of simple molecules, mainly due to the difference between the chain connectivity in polymers and the assemblies of simple 
molecules (Doye \& Frenkel 1999). It was established in the literature that this not only affects the equilibrium crystal structures but also the kinetics of crystal growth (Mandelken 2001).

A well-known description of crystallization kinetics is the heuristic Avrami phase transition theory (Avrami 1939). This was initially based on the assumptions that nucleation occurs randomly and homogeneously over the entire untransformed portion of the material, and that growth rate does not depend on the extent of transformation, yet is the same in all directions. This was mainly adapted from the formulations intended for metallurgy to the needs of polymer crystallization. Later these were simplified for application to bulk crystallization of polymers (Evans 1945; Meares 1965). Also called as JMAK equation (Johnson-Mehl-AvramiKolmogorov equation), this was reduced to a form that gives the fraction of transformed material after a hold time at a given temperature and is expressed as:

$$
\ln (-\ln [1-Y(t)])=\ln K+n \ln t,
$$

$K$ and $n$ obtained from the plot of $\ln (1 /(1-Y))$ versus $\ln (t)$. The details on nucleation and growth effects, and the work carried out and reported in the literature is given in the following sub-sections.

\subsection{Primary and secondary nucleation}

In an unseeded crystallization process, primary nucleation triggers the formation of first formation of crystals, and remains the main nucleation mechanism throughout the entire process, due to the high supersaturation values. The nucleation detection technique is known to affect the measured MSZW. Numerous techniques such as electrical conductivity, ultrasound velocity, Focussed Beam Reflectance Measurement (FBRMs), and turbidity have been reported in literatures which were employed to detect nucleation, figure 4 shows a schematic of the primary and secondary nucleation phenomena. Primary nucleation herein is also present due to the presence of an external seed material. The secondary nucleation is shown to occur as aggregation, attrition/ breakage and abrasion processes. Mostly, attrition or breakage occurs in dilute solid suspensions, while abrasion is most common in concentrated solid suspensions. Secondary nucleation occurs mainly due to the collision of larger particles with the crystallizer internals or with other crystals leading to formation of crystal fragments and further attrition leading to growth of these crystal fragments. This is most dominant in most of the continuous crystallization processes. This type of nucleation is dependent upon the fluid dynamics at larger as well as the smaller length scales, and hence on the volume and the operating conditions of the crystallizer. As fluid dynamics

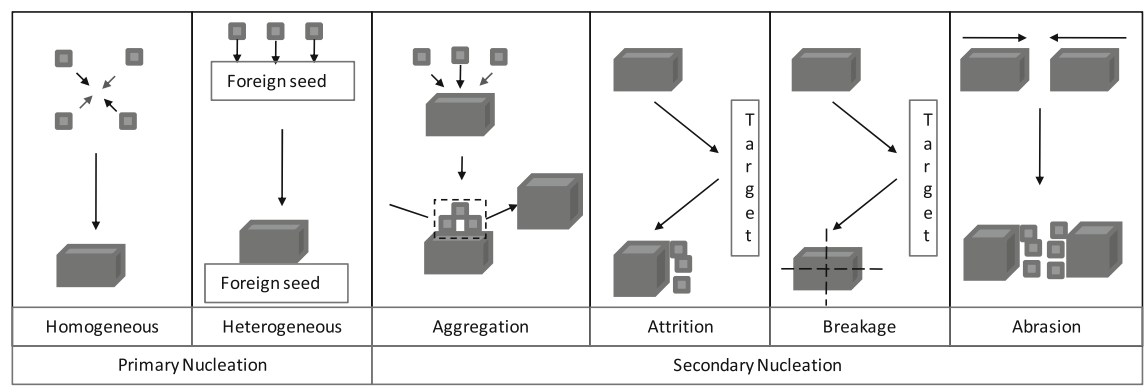

Figure 4. Schematic of primary and secondary nucleation phenomena. 
varies differently under various operating and geometry conditions, power law description for secondary nucleation does not hold good (Kramer \& Jansens 2003).

The mechanisms which govern the primary and secondary nucleation are different and hence lead to different rate expressions. No matter whether a crystallization system is continuous or batch and whether it employs evaporation, indirect cooling or anti-solvent addition, the main source of crystal nuclei is normally secondary (i.e., nucleation resulting from existing crystals). Secondary nucleation requires the presence of "parent" crystals that are involved in the initiation of further nucleation by three mechanisms: breakage, attrition due to collisions, and removal of semi-ordered surface layers through fluid shear and collisions. It takes place on crystal surfaces and is due to crystal-to-crystal (CC), crystal-to-impeller (CI) or crystal-to-wall (CW) contact. The magnitude of secondary nucleation is significantly influenced by the level of supersaturation within the crystallizer. In contrast to macro attrition/breakage, the crystals are not broken into smaller pieces. Both growth and nucleation compete for the new solute as it comes out of solution. Second, secondary nucleation can also originate due to the solute being in the liquid state, such mechanisms include nucleation due to fluid shear and impurity concentration gradient nucleation (Myerson 2002). Two contrasting approaches can be found in the literature for the evaluation of secondary nucleation kinetics for a given solution system. The first and older approach is through the measurement of meta stable zone width (MSZW) and induction times for nucleation in the presence of seed crystals. The presence of seed particles in solution is known to decrease the measured MSZWs and induction times, when compared to unseeded solutions (Kubota 2010). Another approach involves the simultaneous evaluation of secondary nucleation and other process kinetics, such as growth rate kinetics, from the experimental data of seeded batch experiments (Worlitschek 2003) and for polymorphic transformations (Scholl et al 2006). Frawley et al (2012), developed a numerical model utilizing the population balance equation (PBE) and method of moments to describe the seeding process, accounting for primary and secondary nucleation, and subsequent crystal growth. They found that secondary nucleation rate was dependent upon seed loading and size fraction of the seed crystals utilised. Secondary nucleation rates were observed to increase with seed surface area, supersaturation and agitation rate. However, the secondary nucleation rate was found to be independent of solution temperature in the temperature range they investigated. Seed loading has also been shown to have a significant effect on inhibiting the occurrence of secondary nucleation events. The mechanism of secondary nucleation was attributed to the detachment of irregular or dendrites from the surface of the added seed crystals, observed by previously for other solution systems.

In the polymer crystallization, based on whether any second phase, such as a foreign particle or surface from another polymer, is present in the system, the nucleation is classified as homogenous nucleation ('primary nucleation') or heterogeneous nucleation ('secondary nucleation'). In primary nucleation, creation of the stable nucleus by intermolecular forces orders the chains in a parallel array (Yang et al 2005). As the temperature goes below the melting temperature, the molecules tend to move toward their lowest energy conformation, a stiffer chain segment, and thus favouring the formation of ordered chains and thus nuclei. Facilitating the formation of stable nuclei, secondary nucleation is also involved at the beginning of crystallization through heterogeneous nucleation agents, such as dust particles. Following nucleation, crystals grow by the deposition of chain segments on the nucleus surface. Due to the long-chain nature, subsequent entanglements, and particular crystal structure, polymers crystallized in the bulk state are never totally crystalline, and a fraction of the polymer is amorphous. Yang et al (2005) investigated, if the Avrami equation (established by a more fundamental approach to crystallization) actually incorporates homogeneous and heterogeneous nucleation, uneven growth of crystals into a particle size distribution, and final Ostwald ripening of the CSD. They adapted the 
distribution kinetics model of nucleation, growth, and aggregation that results in an S-shape curve of crystallinity versus time, to explore the polymer crystallization. They concluded that the population dynamics can well explain the combined processes of nucleation and growth. The details on population balance models and solution methods are described in section 5.2.

\subsection{Ostwald ripening}

Ostwald ripening involves dissolution of crystals into smaller ones and their adherence to larger crystals. This thermodynamically-driven spontaneous process occurs because larger particles are more energetically stable than smaller particles (internal pressure inversely proportional to the radius of the particles). This stems from the fact that molecules on the surface of a particle are energetically less stable than the ones already well ordered and packed in the interior. Large particles, with their lower surface to volume ratio, results in a lower energy state (and have a lower surface energy). As the system tries to lower its overall energy, molecules on the surface of a small (energetically unfavourable) particle will tend to detach and diffuse through solution and then attach to the surface of larger particle. Therefore, the number of smaller particles continues to shrink, while larger particles continue to grow. For crystallization, combined aggregation and monomer addition (growth) is often called agglomeration. The reverse is dissolution (monomer removal) and breakage. Solution concentration, either supersaturated or undersaturated, drives the reversible growth or dissolution, respectively. The relative saturation, or degree of non-equilibrium, is determined by thermodynamics, principally the Gibbs-Thomson relationship for interfacial energy. Smaller particles have a higher fugacity and are more soluble. Larger particles are less soluble and thus tend to grow at the expense of smaller particles, leading to Ostwald ripening. Tavare (1987) attempted the simulation of Ostwald ripening process in a perfectly mixed reactive batch precipitation process.

Ripening models are based on the Kelvin or Gibbs-Thomson equation, which gives the relationship for the ratio of interfacial energy to thermal energy, such that smaller clusters are less stable than larger ones and therefore larger clusters grow at the expense of smaller ones. The Kelvin equation for a given supersaturation provides an expression for the critical nucleus size, above which the cluster grows, below which the cluster is unstable and dissolves unless fluctuations allow homogeneous nucleation (Adamson \& Ghast 1997). Clusters become more soluble as they become smaller, and gradually disappear, yielding up their mass for growth of larger clusters. The ultimate state is reached when but one cluster remains in equilibrium with the monomer solution. Although most papers on the theory of ripening mention the reduction in cluster numbers during ripening, this effect is sometimes neglected altogether. The final equilibrium condition of one large cluster is attained when de-nucleation is considered.

Some models do also focus on the mass transfer rate for dissolution and growth. Conventional theories are usually based on first-order differential equations for cluster growth of the cluster size distribution (Marqusee \& Ross 1983). The growth rate is represented by the difference between the solution concentration and its equilibrium saturation value as the driving force. When supersaturation is much greater than unity, homogeneous nucleation can occur, and all stable clusters larger than critical nucleus size will grow. The goal is to describe ripening for a distribution of clusters when supersaturation causes larger clusters to grow while smaller clusters vanish. Some ripening models apply to only a few particles, rather than the more interesting distribution of particle sizes. Typically the exponential in the Kelvin equation is approximated by the first-order linear term. An analytical asymptotic solution is usually sought that applies for long time and is independent of initial conditions. Most theories do not explicitly represent the 
evolution of a single large cluster, which would be expressed as a Dirac distribution. McCoy \& Madras (2008) used distribution kinetics to describe the complete time range for transition from a homogeneous supersaturated phase through the first appearance of nuclei, subsequent monomer deposition and growth, and eventual ripening to a single large remaining cluster (cluster refers to either liquid droplet or a solid precipitate). They concluded that a CSD approach for nucleation accompanied by crystal growth and Ostwald ripening serves to describe quantitatively the crystallization kinetics of polymer melt. Though nucleation and growth kinetics have been studied extensively, this slight demarcation of Ostwald ripening has not been much explored in the available literature.

\subsection{Crystal habit}

Crystal habit or crystal shape is either thermodynamically or kinetically controlled. Suspension density conventionally varies depending on the crystal habit/shape, in response to internal and external factors. In order to steer crystals towards target shape, or away from undesired shape, factors such as solvent type, impurity, or additive concentrations, solution temperature, and supersaturation. Transiently, as the crystallization progresses, crystal habit is rather dependent on the growth process occurring. The edges or corners of a crystal are related to layers of molecules which are accompanied by intermolecular interaction, whereas the layer-by-layer growth associated with a crystal face is related to intermolecular interactions that are less energetic in nature. A solvent or additive molecule which is able to compete for a site at an incoming point associated with the layer-by-layer growth process would be capable of disrupting the magnitude of the intermolecular interactions present between growth layers. This may then lead to a different ranking of strength of interaction between growth layers, which would manifest itself in a change in overall morphology of the crystal. Another factor is the effect of solvent or additive molecules on the growth mechanism, particularly when solubility is affected. As solubility defines supersaturation, any change in supersaturation can cause the growth mechanism to move from screw dislocation to surface nucleation and eventually to continuous growth. This change in growth behaviour was also discussed in literature (Blagden et al 2007).

A number of examples in the literature demonstrate the effects of changing crystal morphology on in vitro dissolution rate, with potential for improving bioavailability. The habit modification by crystallization using different solvents, additives and crystallization conditions has been reported (Dirksen \& Ring 1991; Adhiyaman \& Basu 2006).

\subsection{Crystal growth}

Once nucleation has been achieved, crystal growth dominates, and is the process which leads to the evolution of crystal form of defined size and shape. The key drivers with regard to the shape of the growing crystal are related to the crystal lattice of the molecular solids and the effects of the choice of solvent and additives on the process of crystal growth. As such, crystal growth is a layer-by-layer process, with the evolution of the layers being defined by the crystal packing of the unit cell. The unit cell in turn describes the critical elements of how a specific molecular species has assembled in a crystalline state in three dimensions (Blagden et al 2007). It is the strength of the intermolecular interaction defined within the unit cell which, at the first level, determines which layers dominate the crystal growth process.

The crystal growth rate, $\mathrm{G}$, will be either diffusion or surface-reaction controlled. Diffusioncontrolled growth (of diffusional growth) implies that the growth rate increases as the extent of 
mixing (or slurry velocity) increases, up to the point where surface reaction, or incorporation of the solute into the lattice, becomes the controlling factor. However, the desire to increase the growth rate and enlarge the size distribution via more agitation may be negated by an increase in nucleation. The crystal growth can be expressed in three different levels: macroscopic, microscopic and molecular. At macroscopic level, heat transfer in solution is faster than mass transfer, hence may not be significant unless exothermic or endothermic heats of the actual contents are very high. On the other hand, mass transfer limitations quite often control the crystal growth. Macroscopic concentration gradients influence the crystal surface concentration profile which most likely can cause instabilities at the surface. Microscopic level deals with formation of step bunches, wherein, layers are formed due to decrease in diffusive flux and thus trap the solvent in these layers of the crystal. At molecular level, the growth units mostly diffuse and attach to the crystal. Crucial factor here is the crystal solution interface. Depending upon the growth rates on the faces of the crystalline products obtained, Tung et al (2009), have shown the images of typical crystal growth rates as one-dimensional, two-dimensional and three-dimensional images. The same is shown in figure 5.

Understanding the shapes of crystals on the basis of different crystal growth rates on different surfaces is helpful for the development of crystallization processes. For example, needle-like crystals have much less surface area for growth. Consequently, more seed is required in order to provide enough surface area for crystal growth. On the other hand, rod-like or cube-like crystals have much more surface area for growth. So, effectively less seed is required. Furthermore, it is common to observe that the fastest-growing surface outgrows itself and disappears. Therefore, the apparent release of supersaturation by crystal growth is significantly reduced after a certain point of crystallization. If it occurs during crystallization, the supersaturation will build up and a short burst of nucleation may occur. This will lead to a much wider particle size distribution that is generally undesirable.

It has been reported in the literature that needle-like and plate-like crystals create additional process complications. These crystals are proven to have higher filtration resistance and poorer solid flow characteristics for formulation than cube-like crystals. Therefore, it is highly desirable to grow thicker crystals. To grow "thick" crystals, experimentally, information on finding the best solvent which favours the formation of such crystals is useful. Meanwhile, solvates and hydrates may form in different solvent environments. Chemical forms, such as salt, free base, and free acid, can also be evaluated. Also, control of release of supersaturation and selection of crystallization conditions to enhance crystal growth over nucleation, offers useful information.

3.4a Stages of crystal growth: As explained in the literature (Dirksen \& Ring 1991), the crystal growth can be described to occur in various stages. To begin with, once nucleation occurs, solute is transported from the bulk of the solution to the crystal surface. This is followed by the adsorption of the crystal surface and diffusion over the surface. Thereby, attachment and diffusion occurs at the step site (where two nearest neighbours bond) sequentially. This leads to an integration into the crystal at a kink site (where three nearest neighbours bond). Finally, diffusion of coordination shell of solvent molecules occurs away from the crystal surface, and heat of crystallization is liberated transporting it away from the crystal. In a comprehensive review by them, the dependency of supersaturation and crystal size on crystal growth rates was tabulated for the various mechanisms of crystal growth such as monosurface nucleation, mono/poly crossover point, polysurface nucleation, screw dislocation and bulk diffusion. 


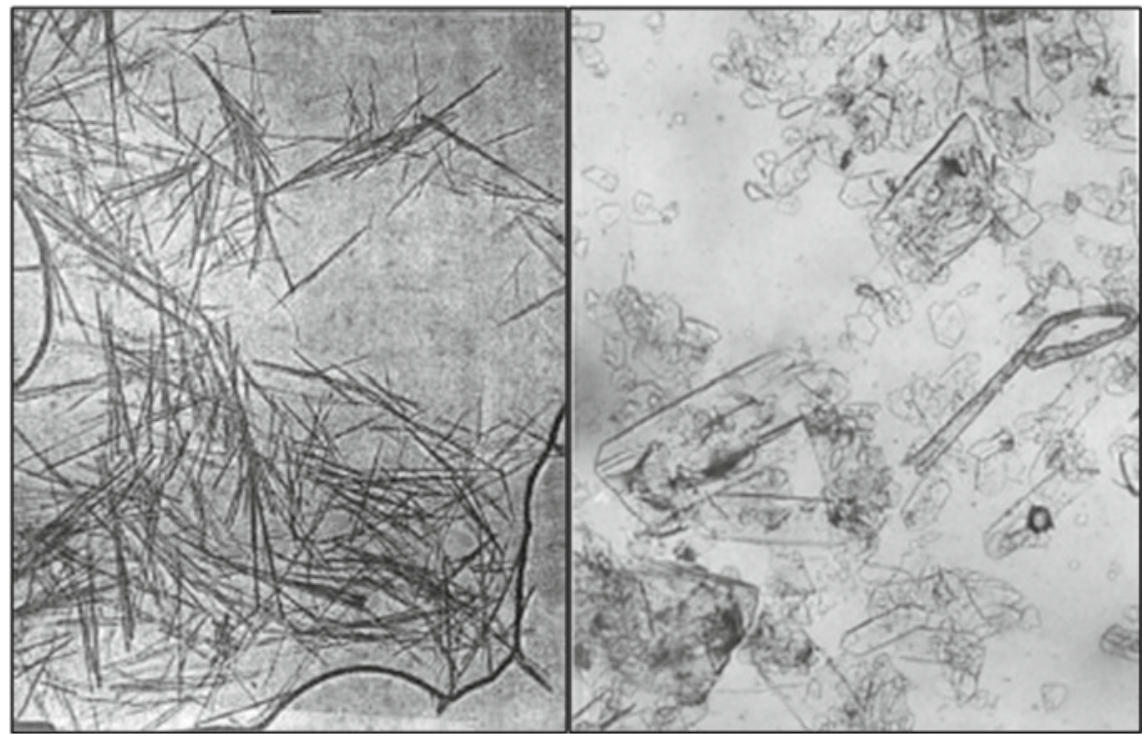

(a)

(b)

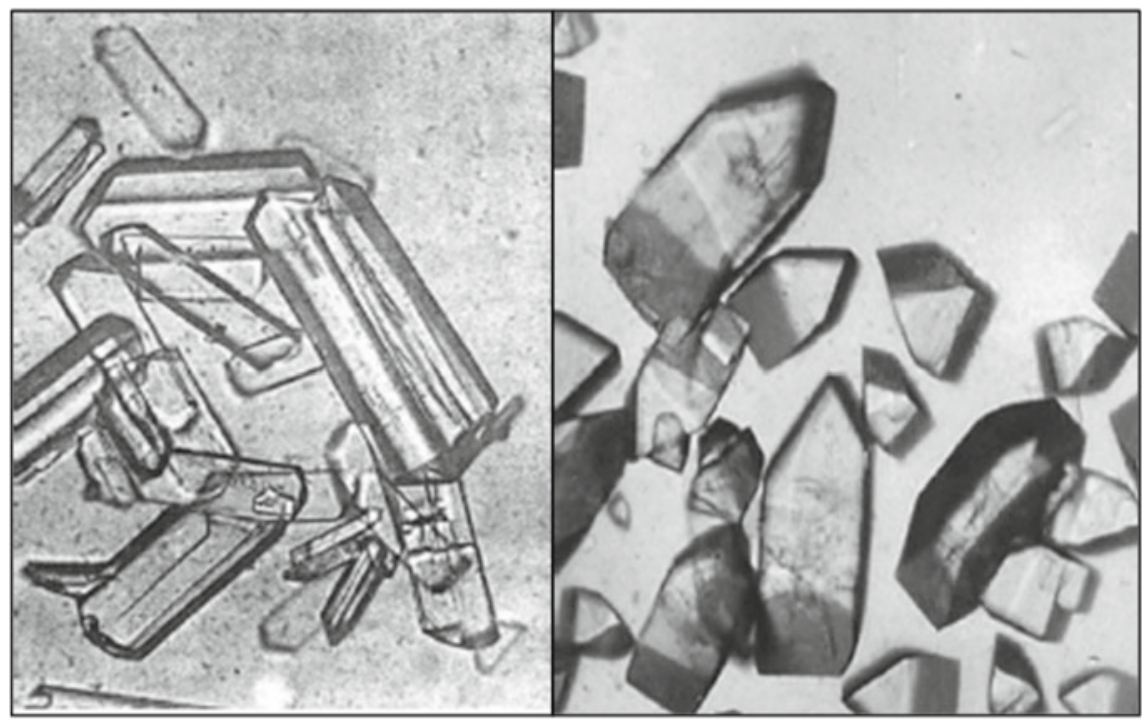

(c)

Figure 5. Images of typical crystal growth shapes (Tung et al 2009). (a) One-dimensional, (b) twodimensional, (c) three-dimensional.

\subsection{Oiling out}

While carrying out a crystallization process, instead of crystallizing from a supersaturated solution, droplets of a second liquid phase were observed to be separated. This phenomenon is frequently termed oiling out or liquid-liquid demixing (Lafferrere et al 2004). It was explained that from a thermodynamic point of view, phase separation normally takes place only when the 
Gibbs free energy of mixing (Eq. (4)) is greater than zero, i.e., $\Delta G_{\operatorname{mix}}>0$. In Eq. (4), $\mu_{\mathrm{A}}^{*}$ and $\mu_{\mathrm{B}}^{*}$ are the reference chemical potentials of components $\mathrm{A}$ and $\mathrm{B}$, respectively.

$$
\Delta G_{\text {mix }}=x_{\mathrm{A}}\left(\mu_{\mathrm{A}}-\mu_{\mathrm{A}}^{*}\right)+x_{\mathrm{B}}\left(\mu_{\mathrm{B}}-\mu_{\mathrm{B}}^{*}\right) .
$$

However in some systems, such as those exhibiting oiling out, phase separation can occur when $\Delta G_{\text {mix }}$ is negative with respect to the unmixed components. As shown in figure 6 for the two component system $\mathrm{A}$ and $\mathrm{B}$ exhibiting oiling out, the net $\Delta G_{\mathrm{mix}}$ is negative for all of their mixtures; however, $\Delta G_{\mathrm{mix}}$ can be minimized at two compositions, $x_{\mathrm{AB}}$ and $x_{\mathrm{BA}}$, such that a miscibility gap is generated. At these compositions, the net overall $\Delta G_{\mathrm{mix}}$ is lower when compared to any mixture between $x_{\mathrm{AB}}$ and $x_{\mathrm{BA}}$, and these are known as the binodal points for mixtures of $\mathrm{A}$ and $\mathrm{B}$.

The key thermodynamic property of the binodal points is that the chemical potential of each species is equal in both phases. Thus mixtures of $\mathrm{A}$ and $\mathrm{B}$ whose compositions are lower than $x_{\mathrm{AB}}$ and higher than $x_{\mathrm{BA}}$ are stable and do not phase separate. Between $x_{\mathrm{AB}}$ and $x_{\mathrm{AB}}^{*}$ and $x_{\mathrm{BA}}$ and $x_{\mathrm{BA}}^{*}$, the system is metastable i.e., phase separation can occur, but an energy barrier has to be overcome to initiate a new phase. The points $x_{\mathrm{AB}}^{*}$ and $x_{\mathrm{BA}}^{*}$ are known as the spinodal points, beyond which the solution is unstable and will spontaneously divide into the two liquid phases of equal chemical potential. This process is termed spinodal decomposition where separation proceeds without any thermodynamic barrier. It should be noted that partial phase immiscibility is a function of temperature. For systems in which the degree of immiscibility decreases with increasing temperature, an Upper Consolute Temperature (UCT) is defined as that temperature at which complete miscibility occurs. In practice oiling out is undesirable since, e.g., the oil phase is often a good solvent for impurities and if it subsequently crystallizes it can render the whole crystallization process worthless in delivering purification. Furthermore, it has been noted that scale-up of such a system will be problematic, since the power criterion of constant power per unit volume will produce smaller droplets due the higher tip speeds of impellers used in larger vessels (Veesler et al 2003). Deneau \& Steele (2005) used various Process Analytical

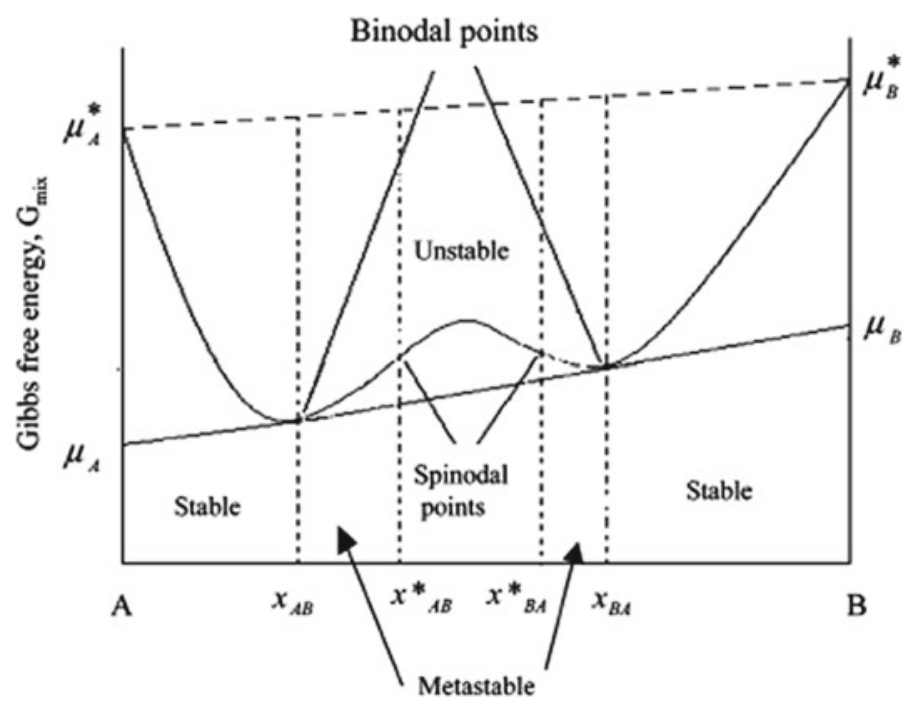

Figure 6. Gibbs free energy function exhibiting liquid-liquid miscibility gap (Deneau \& Steele 2005). 
Techniques (PAT) such as FBRM, PVM and ATR-UV/Vis spectrometry to follow oiling out and crystallization. They found the area between the solubility and oilng curves to be similar to the metastable zone of a cooling crystallization, and that this could be controlled with high seeding loading at all cooling rates.

Kiesow et al (2008), in their experiments and predictions of oiling out used the PerturbedChain Statistical Associating Fluid Theory (PC-SAFT) equation of state to calculate both the liquid-liquid demixing as well as the solubility curve. This was applied to model experimental data of the 4,4' dihydroxydiphenylsulfone-solubility in pure solvents (water, acetone, and 2propanol). In a second step, the model is used to predict the solubility of DHDPS in solvent mixtures (water/acetone and water/2-propanol, respectively) with various solvent compositions as well as the oiling out behaviour without using any additional adjustable parameters. They showed that upon changing the solvent, the oiling out could be prevented, but this was more of a qualitative study. Micrographs of the crystals obtained revealed the influence of the oiling out on the properties of the solid product. Size, shape and degree of agglomeration of the particles vary depending on whether an oiling out occurred during the cooling process or not.

In general, in the case of oiling, supersaturation is in great excess of metastable limit, or the solution is sufficiently concentrated with nucleation inhibiting impurities. As a result the solute forms a liquid phase consisting of solvent-solute concentrate rather than a stable crystalline form. Oils are metastable and may crystallize spontaneously with sufficient holding times. Oiling is often prevented through seeding or by nucleation at low supersaturation; if this approach is unsuccessful, impurities must be individually investigated for their inhibition of nucleation and growth, with the problematic impurity removed by alternative means.

Until recently, there were only a few references in the literature on oiling out, that helped process-chemists and engineers to understand and ultimately control this phenomenon (Bonnett et al 2003; Lafferrere et al 2004; Deneau \& Steele 2005; Kiesow et al 2008; Codan et al 2011).

\section{Hydrodynamic aspects}

\subsection{Solid-liquid suspensions}

Suspension of solid particles in a liquid is one of the most important unit operations in process industries. Solid suspensions are typically carried out in mechanically agitated or stirred vessels. Pumped liquid jets have also been used to suspend low concentrations of relatively slow settling solids. Although static mixers have been used to disperse fine solids into polymers, application of the technology is limited (Paul et al 2004). Mechanically agitated solid-liquid contactors are most frequently used for the hydrometallurgical operations, crystallization, precipitation, dissolution, ion exchange, adsorption, slurry polymerisation etc. Reliable design of stirred reactors used in solid-liquid suspensions requires an in-depth understanding of solid-liquid suspension which is highly impacted by impeller generated flow, turbulence, solids loading and so on. Though, these have been dealt with, in various research works, an accurate and quantitative determination of suspension quality is still an important parameter to study.

Conventionally, the primary objectives of solid-liquid mixing are to create and maintain slurry and/or to promote and enhance the rate of mass transfer between the solid and liquid phases. Figure 7 displays the operations and the processes promoted by the solid-liquid mixing. These unit operations, with the exception of dispersion, involve mass transfer between the solid and liquid phases. Solid suspension requires the input of mechanical energy into the fluid-solid system 


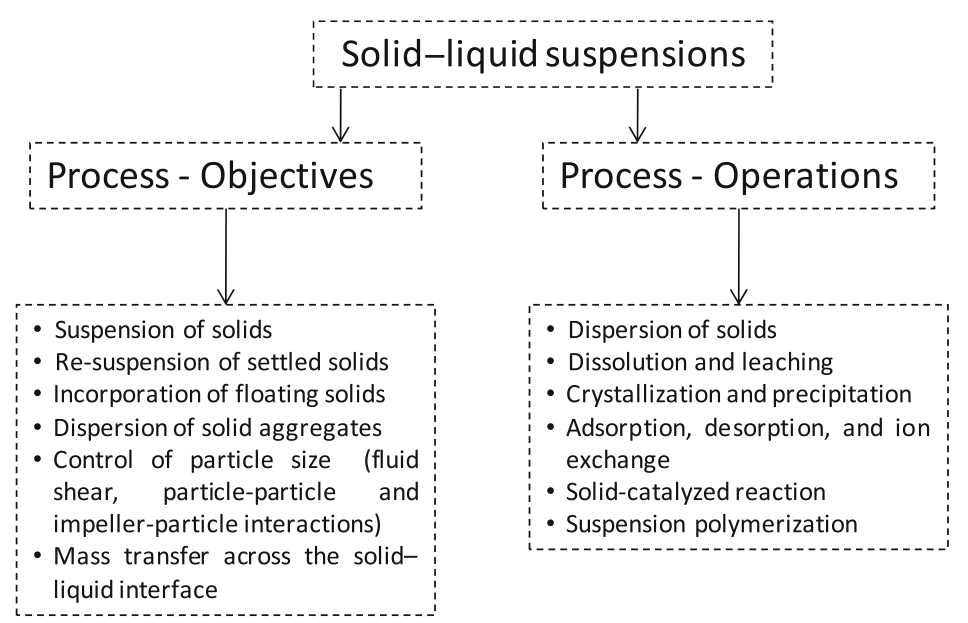

Figure 7. Operations and processes promoted by solid-liquid mixing.

by some mode of agitation. The input energy creates a turbulent flow field in which solid particles are lifted from the vessel base and subsequently dispersed and distributed throughout the liquid. Studies in early 80 s until late 90 s discussed in detail confirmation of the complex hydrodynamic interactions between solid particles and the fluid through experimentation (Nienow 1985; Guiraud et al 1997). The magnitude of free settling velocity also has proven useful in characterizing solid suspensions. This velocity, occurs when the drag force balances the buoyancy and gravitational force of the fluid on the particle. In an agitated solid suspension, because of the complex turbulent hydrodynamic field, including solid-solid interactions, it is difficult to clearly define and/or measure a particle settling velocity. However, the particle settling velocity in an agitated solid suspension is a function of the free settling velocity and is always less than the free settling velocity (Guiraud et al 1997).

The quality of solid suspension can be broadly divided into three regimes, i.e., on-bottom (partial), off-bottom (complete), and homogeneous (uniform) suspension regimes. The distinct level at which most of the solids get lifted within the fluid where the solid-liquid interface appears, is called the "cloud height". Extensive data on measurement of cloud height within the stirred vessel was carried out, shown in the literature, thus providing a qualitative indication of suspension quality. There existed some discrepancies in modelling studies on the variation in increase of cloud height with impeller speed. Sardeshpande et al (2009) characterized solid-liquid suspension based on cloud height, power consumption, liquid phase mixing and circulation time for solid loadings upto $7 \% \mathrm{v} / \mathrm{v}$. The same work also indicated various suspension regimes based on the cloud height.

It has also been pointed out in the recent literature that clear-cut methods for estimating power requirements for solid-liquid stirred tanks are yet unavailable. The general approach usually adopted in the available literature makes use of the available correlation for a single phase power number, $N_{\mathrm{p}}$ (based on liquid density), and for a two phase power number, $N_{\mathrm{p}}$ (based on liquid density), or $N_{\mathrm{ps}}$ (based on slurry density) to predict power requirements in slurry systems. Range of experimental data was covered in the studies reported on power dissipation and its effect on solids concentration, impeller geometries, and particle size. On the whole, reported studies showed scattered information about effect of key parameters on power consumption in a solidliquid slurry system (Rewatkar \& Joshi 1991; Pinelli et al 2001; Sardeshpande et al 2011). 


\subsection{Classical design aspects}

Conventional design and scale-up aspects based on the similarity concept are decided based on geometric, kinematic and dynamic similarities. These details have been amply covered in the literature for the cases of stirred vessels. In order to summarize the classical design aspects, figure 8 has been framed depicting the importance of corner stones needed in design of solidliquid suspensions.

To design an effective stirred tank, an efficient impeller should be chosen for the process duty. More than one impeller may be needed for tanks with high aspect ratio $(\mathrm{H} / \mathrm{T}>1.5)$. Sizing of the impeller is done in conjunction with mixer speed to achieve the desired process result. The appropriate size and type of wall baffles must be selected to create an effective flow pattern. The mixer power is then estimated from available data on impeller characteristics, and the drive size is determined. The mixer design is finalized with mechanical design of the shaft, impeller blade thickness, baffle thickness and supports, inlet/outlet nozzles, bearings, and other support structures (figure 9).

4.2a Critical suspension speed: Conventionally, solid suspension in stirred reactors is characterized by the just enough speed of the impeller required to keep the solids suspended off the bottom. Though this concept dates back to early 60s, it is still considered one of the crucial parameters for design of stirred reactors needed for solid-liquid suspensions. The complete offbottom suspension ensures the minimum speed of agitation at which all particles are completely suspended $\left(N_{\mathrm{js}}\right)$ and hence majority of solid surface area becomes available for the chemical reaction, heat and mass transport, etc. This is most often expressed in dimensionless form as:

$$
N_{\mathrm{js}}=S \nu^{0.1}\left[\frac{g\left(\rho_{\mathrm{s}}-\rho_{\mathrm{l}}\right)}{\rho_{\mathrm{l}}}\right]^{0.45} x^{0.13} d_{\mathrm{p}}^{0.2} D^{-0.85} .
$$

$D$ is the impeller diameter, $d_{\mathrm{p}}$ the mass mean particle diameter, $X$ is a mass ratio of suspended solids and liquids; $S$ is a dimensionless number based on the impeller type. As the impeller speed is increased beyond the critical speed, the solid concentration becomes more uniform. In

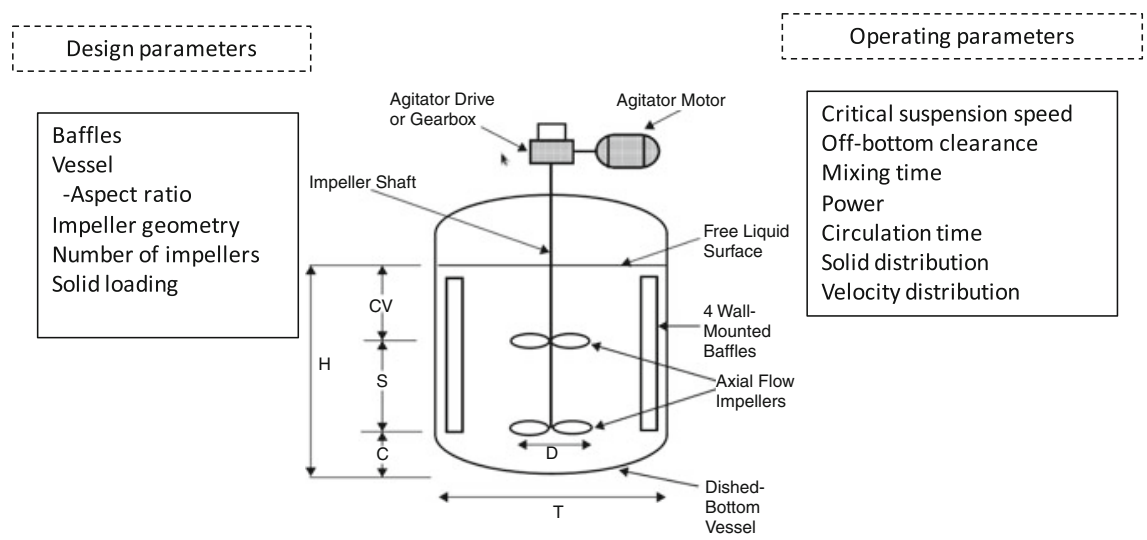

Figure 8. Classification of design and operating parameters for solid suspensions in mechanically agitated vessels. 
Commercial crystallizers for laboratory scale applications

Ni-tech-Continuous oscillatory baffled reactors

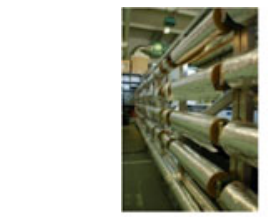

Syrris - Reactor Master

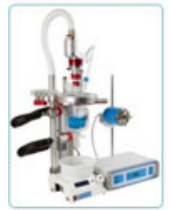

Mettler-Toledo - (a) Labmax, (b) Optimax, (c) Easymax
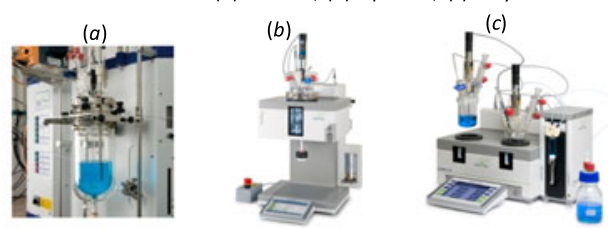

Aventium Crystal-16

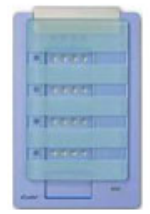

Figure 9. Few of the laboratory scale commercial crystallizers available in industrial market.

homogeneous suspension state, the increase in speed above the just suspension generates more uniform distribution of solids along the reactor height. However above the just suspension speed, the rate of mass transfer increases very slowly. This implies that the rational design as well as reliable operation of this equipment depends greatly on the understanding of their complex fluid dynamics, which includes solids suspension and distribution, power consumption, mixing characteristics, etc. Variety of equations for the prediction of just suspension speed and large number of techniques for the measurement of solid concentration profiles are available in the literature. However, nearly all of them are derived from experimental results carried out in stirred vessels of small size (Joshi et al 2011).

\subsection{CFD modelling}

The description of fluid dynamics in stirred vessels is well addressed in the literature, including the usage of commercial softwares, especially in single phase flows. The use of computational fluid dynamics (CFD) codes is suitable for the simulation of crystallizers that are not perfectly mixed, since in this case the simulation is best handled by solving the complete transport equations (Sha et al 1999). Since all mathematical details of CFD models and Population Balance Models, including the equations, are well covered in the literature, they have not been described here to avoid redundancy. CFD codes use either finite elements or finite volume methods in which the conservation equations are applied directly to sub-regions to obtain numerical values for the variables of importance (Koenig 1998). While such codes should probably be applied in the design of any industrial scale crystallizer, the computations are rather intensive for such simulations to be used for the development of estimation and control algorithms.

Gosman et al (1992) calculated the solid-liquid flow in a stirred vessel with a three dimensional calculation domain. The Eulerian-Eulerian model was adopted and the drag force as well as the added mass force were taken into account for describing the momentum exchange between phases. The impeller region was treated as a "black box", and the experimental data were imposed on the boundary of the volume swept by the impeller blades as the necessary boundary conditions. The predictions have been moderately compared with the experimental 
data. Further, they have carried out sensitivity analysis to understand the influence of the various interphase momentum transfer forces. It has been found that only drag force is important. Later, some extensions were presented to calculate the spatial distribution of solids (Bakker \& van den Akker 1994). Micale et al (2000) predicted the vertical particle concentration profiles for single and multiple impeller configurations using commercial softwares. Only the drag force was considered using the pinneli drag model and the turbulent transport of the particles as well as the turbulence modification by the particles was neglected. Joshi et al (2011) pointed that the main drawback of the above drag model could yield an ever increasing drag when the particles become larger. This is physically not correct since the standard drag coefficient is not recovered even for extremely large particles. This was attributed to the fact that the added mass was not considered. Studies were also reported on different physical effects relevant to particle transport, where in, effects of impeller speed, clearance, particle size were focused. Effect of turbulence on drag, particle turbulent distribution was also reported for systems with low particle volume fraction (Ljungqvist \& Rasmuson 2001; Sha et al 2001; Montante \& Magelli 2005; Khopkar et al 2006; Sbrizzai et al 2006). Various attempts have been reported on the behaviour of dense suspensions inside stirred tanks showing a good agreement between experiment and numerical predictions for steady-state systems and also on the study the influence of drag force on solid suspension and distribution (Micale et al 2004; Montante \& Magelli 2005; Khopkar et al 2006). Joshi et al (2011), presented a comprehensive review on CFD modelling studies in stirred tanks, stating that it is worth noting that dense suspensions require a correct modelling of both solid-liquid and particle-particle interactions. Several studies do highlight the importance of correctly describing the interaction between phases by specifying interphase momentum exchange terms such as Basset force, virtual mass force, lift force and drag forces.

Guha et al (2007) investigated a solid-liquid suspension in a baffled tank stirred by a radial impeller with an overall solids holdup of $1 \%(\mathrm{v} / \mathrm{v})(2.5 \%, \mathrm{w} / \mathrm{w})$. Authors compared, with relevant experimental data, the CFD results obtained either by Large Eddy Simulations (LESs), based on a Eulerian-Lagrangian framework, or by a standard Eulerian-Eulerian approach. It was stated that, in such dilute conditions, LES approach provides reasonably better agreement in predicting the tangential component of the azimuthally averaged velocities and the turbulent kinetic energy.

Kramer \& Jansens (2003), in the review, offered a detailed explanation on the application of fluid dynamic modelling to industrial crystallizers. CFD has been used to model reactive semibatch crystallization and has been used to predict the effect of mixing on the supersaturation distribution and the resulting size of the crystals (Wei et al 2001; Baldyga \& Orcuich 2001). Zauner \& Jones (2002), used CFD in conjunction with crystallization kinetics and solubility data to simultaneously solve the mass and population balances for a reactive crystallization, allowing the impact of feed rate, agitation intensity, feed point and feed tube diameter on nucleation rate and crystal size to be illustrated. CFD has also been used to study the impact of mixing on the product yield of crystallization (Akiti \& Armenante 2004).

\section{Crystallization control, design and scale-up}

Chemical aspects, such as solubility and polymorphism, play a key role in the initial design of a crystallization process. Achieving the correct crystal morphology is one of the main goals of a crystallization process design. The second main goal is to deliver a robust process scaleup from laboratory to pilot plant and manufacturing scale to minimize variability in product 
quality between batches. To achieve this, it is essential to understand the chemistry and related information such as solubility and kinetics.

The design is mainly influenced by the interaction between the kinetics and the fluid dynamics which affect the crystal size and formation. At large length scales, the fluid dynamics determine the transport and circulation of slurry inside the crystallizer, and whether or not the slurry is well mixed and suspended. In larger crystallizers, non-uniform distribution in process conditions such as temperature, supersaturation and turbulence are unavoidable. This affects both primary and secondary nucleation. Hollander et al (2001) showed that this type of inhomogeneity can have a great impact on product quality depending on the volume of the equipment. At smaller length scales, the flow field induces collisions of the crystals with the crystallizer internals which leads to secondary nucleation by attrition of larger particles. Finally, at the smallest scales, the turbulent flow is responsible for the crystal-crystal collisions and the mass transport of the bulk of the solution to the crystal surface. Hence Bermingham et al (2000) concluded that in order to take the local effects into account, compartmentalization of the crystallizer is mandatory. It was further emphasized in the work of Bermingham et al (2000), that for evaporative or cooling crystallization, wherein secondary nucleation and growth are more dominant, the number of compartments can be limited from about a few up to a tenfold. In case of precipitation and antisolvent crystallization, the selection of number of compartments is based on the geometry of the hardware, fluid dynamics in the crystallizer, rates of kinetics and their sensitivity to local changes.

Kramer \& Jansens (2003) presented a review on industrial crystallizers, bringing out tools for design and scale-up of industrial crystallizers. Scale-up of cooling crystallization process, is mainly dependent upon maintaining a cooling profile (beyond a threshold rate), while managing the wall temperature and the mixing which impacts both heat and mass transfer. In case of antisolvent crystallization, along with the temperature gradients occurring due to the anti-solvent addition and mixing, the anti-solvent addition rate plays a major role. While, in the case of reactive crystallization, the rate of addition of reagent and heat of reaction is crucial.

The scale-up of crystallization processes represents one of the most complex tasks in process engineering as usually a number of target quantities have to be kept within narrow boundaries, such as yield, selectivity, purity, and often particle size distribution. The limit on number and scale of experiments is normally compensated by the use of computational engineering tools based on CFD and dynamic process modelling. The development of methods related to these tools, including the numerical solution of differential equations, goes back as far as the late 1950s. Yet, only in the past 2-3 decades, due to great advances in microprocessor technology, enough calculating power at reasonable cost had been made available to a wider user base. This has extended the application to further fields of commercial interest, including the flow and reaction profile within stirred tank reactors in the chemical and pharmaceutical industry. Since then, the use of CFD and process modelling tools has steadily increased from simulating simple single phase flows and reaction kinetics to assessing multiphase systems with complex kinetic profiles.

For appropriate selection of scale-up parameters, crystallization scale-up involves understanding of a system's relative sensitivities to heat and/or mass transfer (Schmidt et al 2004). During scale-up however, physical effects can become equally important for the success of a campaign as the underlying chemistry itself, as is shown in the two case studies below. For example, particle size and the particle size distribution (PSD) can be influenced through settling, attrition, agglomeration, and local flow conditions. These effects are based on parameters directly linked to the concept of mixing, such as power input per unit volume, suspension, shear, and heat transfer, all of which can be investigated using CFD software and which must be considered on scale-up. Such an investigation however, only leads to qualitative or, if combined with 
integral scale-up rules and experimental investigations, to semi quantitative results. To achieve a fully quantitative scale-up, intrinsic kinetic data have to be collected and correlated with the reactor setup and geometry via CFD software. However, this method is very time, material, and resource intensive and relies on the ready availability of full experimental data sets. Especially in the fine chemical and pharmaceutical industry, this desirable situation is far from reality as there are often severe constraints in these areas. Therefore, a method is presented on how these limitations can be overcome by applying a semi-quantitative approach based on the combination of experimental work, the application of integral scale-up rules and CFD/process modelling tools and last but not least, process experience.

For the successful application of scale-up rules based on mixing concepts of power input per unit volume $(\mathrm{P} / \mathrm{V})$, suspension, shear and heat transfer, it is important to ensure geometrical similarity between the crystallization vessels, including impellers and baffles, from laboratory to pilot and manufacturing plant scale. Each of these quantities requires a different scale-up criterion depending on stirrer speed, and ideally, all of them should be kept constant on scale-up. However, keeping one criterion constant always violates another. For example, a certain stirrer speed, determined by maintaining the suspension conditions constant on scale-up, might be ideal to suspend particles in a specific process at large scale but might at the same time lead to crystal attrition due to an increase in tip speed. This would then lead to the creation of fines with adverse effects on downstream processing. It is therefore important to perform laboratory experiments to determine the main influencing parameters and associated scale-up factors. CFD models can then be applied to verify the obtained experimental and calculated results of a projected process scale-up.

Under ideal conditions, scale-up leads to identical levels of supersaturation in all equivalent regions of the laboratory, pilot and plant units, such that the CSD and crystal purity are optimized. In other words, the driving force for crystallization would be the same at all scales, with good mass transfer from the bulk solution to the growing crystal surface. In addition, the slurry densities and mixing times would be the same, contact times between the crystals and supersaturated liquor would be equal, shear forces imparted by the agitator to the slurry would be equal, the Reynolds numbers $\left(N_{\mathrm{Re}}\right)$ and Froude numbers $\left(N_{\mathrm{Fr}}\right)$ of the fluid would be identical, and the contact frequency and energy between crystals, as well as between the agitator and crystals, would be the same. However, these goals are often conflicting, rendering it next to impossible to attain the same degree of supersaturation or to replicate mixing parameters upon scale-up, even with geometric similarity. In fact, geometric similarity assures that equivalency of all parameters will not be achieved. McKeown et al (2011) gave a detailed graphical representation of the frequently used crystallization design modes in pharmaceutical processes, showing the various stages starting from dissolution to the completion of crystallization.

\subsection{Polymorphism}

Polymorphism occurs when a single compound exists in two- or more solid forms that have identical chemical structures but different crystal lattice structures. These forms are significant, as they differ in properties such as solubility, melting point, dissolution rate and bioavailability, chemical and physical stability, habit and associated powder properties. This clearly identifies that the desired form needs to be defined prior to the crystallization design. While designing crystallization, it is often desired to produce the crystalline form that is most stable at the solution composition and temperature of isolation. If the form being produced is not thermodynamically stable, it is possible for form conversion to occur at some point in the life cycle of the product, 
with the unstable form becoming difficult or nearly impossible to manufacture. Since the conversion from an unstable to a stable form is a kinetic process, it can be affected by changes in impurities, equipment, concentration and other process variables.

Rapid changes of crystal shape can also be induced by changes in solvent or solvent mixture (Winn \& Doherty 2000) and by the presence of quite small amounts of surface active impurities in solution that act as growth inhibitors for certain crystal planes. Growth inhibitors may be added deliberately to modify the crystal shape, or may be present as a result of the manufacturing conditions (e.g., due to reaction chemistry). An example of this is the case of paracetamol crystallization, whereby small amounts of reaction by-products, such as metacetamol, change the paracetamol crystal shape from equant to needle-like. Changes in supersaturation may also induce changes in shape, but this is typically not as dramatic (Shekunov \& Grant 1997). Consequently, the potential for engineering changes in crystal shape is enormous, although this is an area that has not been as extensively cultivated by pharmaceutical industry (Variankaval et al 2008). Figure 10 presents an instant in time during the solvent-mediated conversion of orthorhombic paracetamol (needles) to the monoclinic form, which exists as prisms and plates in benzyl alcohol (presented in their work). More commonly, the growth inhibitors that are at play in API crystallizations are naturally occurring by-products of upstream chemical steps, and there is generally limited capacity to predict the extent to which low-levels of these impurities may alter crystal habit.

ter Horst et al (2002), presented a modelling procedure for the prediction of interfacial energies between two polymorphs and solvents for the mode compound HMX (cyclomethylene tetramine), known to exist in three different polymorphic forms. Statistically 85\% APIs exhibit polymorphism (pseudo) and 50\% have multiple forms. The capability to reliably manufacture specific stable polymorphs is an integral part of API development process. This is accomplished by a thorough and systematic process involving the polymorph discovery stage, polymorph detection and analytical characterization of the properties of the form(s) discovered (Karpinski 2006). In the same work, various approaches such as XRPD, Raman, DSC, TGA/DTA, DVS,

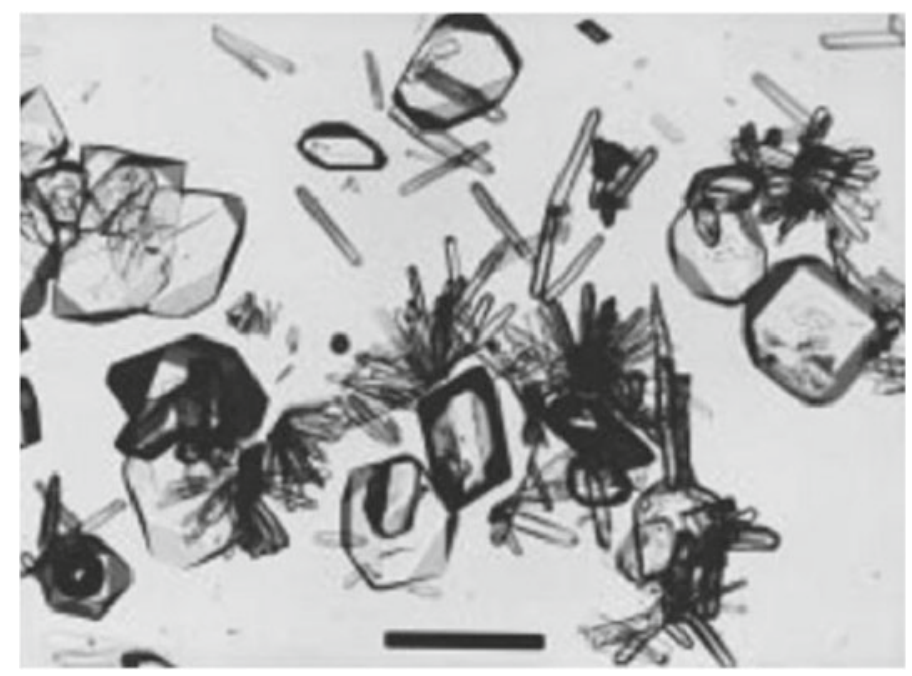

Figure 10. Solution mediated conversion of orthorhombic paracetamol to monoclinic paracetamol in benzyl alcohol (Variankaval et al 2008). 
FT-IR, hot-stage microscopy and SEM were used. It was previously established that IR spectroscopy was restricted to binary mixtures, while XRD and Raman spectroscopy have the possibility to discriminate three different phases. He showed how Raman spectroscopy associated with a multivariable regression, such as partial least squares method established from tertiary calibration, becomes an accurate analysis for tertiary polymorph mixtures of calcium carbonate. However, it was also mentioned that the accuracy of quantitative determination can vary with the chemical system studied. The advantage of polymorphism is that the chemical identity remains unaltered from one polymorph to another, such that a direct correlation between the activity and solid state structure may be made. Polymorphs are categorized into two types, monotropes and enantiotropes, depending upon their stability with respect to the range of temperatures and pressures. If one of the polymorphs is stable (i.e., has lower free energy content and solubility) over a certain temperature range and pressure, while the other polymorph is stable over a different temperature range and pressure, then the two polymorphs are said to be enantiotropes. On the other hand, when only one polymorph is stable at all temperatures below the melting point, with all the other polymorphs being unstable, they are termed as monotropes (Purohit \& Venugopalan 2009). This work also reports a comprehensive review on polymorphism and their characterization. They offered several examples of ploymorphs, detailing on how each molecular arrangement leads to different nuclei, onto different aggregates and to different polymorphs.

Stable polymorph formation via polymorph transformation can be followed if an unstable polymorph is crystallized first with spontaneous nucleation. Some crystals are unstable also as a dry solid, but many unstable forms in the solutions are stable when they are dry crystals (Mullin 2001). The crystallization process can be used to control polymorph formation by adjusting the temperature range, cooling rate or supersaturation level in general, the used concentrations, seed crystals, and additives (Ohtaki 1998; Brittain 1999).

\subsection{Crystal Size Distribution (CSD)}

As a crystalline product with uniform shape and size is desirable, the CSD determines the efficiency of various downstream processes. Control on particle size distributions is becoming increasingly important in chemical and pharmaceutical industry. In many pharmaceuticals applications, micron sized particles are preferred. Recent techniques such as Rapid Expansion of Supercritical Solutions (RESS), Supercritical Anti-Solvent (SAS/PCA Precipitation with Compressed Anti-Solvent) and Gas Anti-Solvent (GAS) are used in pharmaceutical industry to obtain desired particle size distribution.

In the RESS process the non-volatile solute of interest is first dissolved in the supercritical fluid. The solution is expanded through the nozzle at mostly supersonic velocities in order to reduce its density and solvent power thus aiding the solute to precipitate in the form of small particles ranging from few microns to hundreds of microns (Tom \& Debenedetti 1991). Advantages of RESS are the production of virtually contaminant-free particles and the relatively wide range of product mean particle sizes that can be obtained. The main limitation is due to the low solubility of most pharmaceutical compounds in carbon-di-oxide even at high pressures. This cannot be overcome by adding a modifier since it would spoil the environmentally benign nature of RESS. Therefore, the maximum productivity achieved by RESS processes is in many cases too small for industrial applications. So far, RESS technology has been applied to precipitation of pharmaceutical substances, proteins, polymeric support materials for controlled drug delivery systems, inorganic solids, various organic and polymeric model systems. Although all these 
applications cover a wide range of promising applications, very few studies have been focused on deeper understanding of this technique to achieve a quantitative description of the process.

Precipitation with Compressed Anti-solvent (PCA) and GAS recrystallization are complementary to RESS, since they exploit the low-solubility of pharmaceutical solvent in a supercritical solvent. The latter uses carbon-di-oxide as anti-solvent for the solute which is initially solubilized in a conventional organic solvent. Upon mixing with carbon-di-oxide, a reduction of the solvent power of the solution occurs and the solute precipitates. This can be achieved in two different ways: in the PCA process, the initial solution is sprayed into compressed carbon-dioxide through a nozzle or capillary. In some cases, two-fluid coaxial nozzles for solution and carbon-di-oxide are used in order to enhance mass transfer efficiency. In the GAS process the initial solution is kept in a vessel and gradually expanded by adding compressed $\mathrm{CO}_{2}$ (either sub-critical or super-critical). Even though PCA is supposed to be generally more promising than GAS recrystallization in terms of production rates, a thorough comparative analysis of the advantages and drawbacks of the two techniques has not been covered in the literature yet (Bungert et al 1998).

PCA has been used to form different polymeric particles such as microspheres and microballoons, fibres and microcellular foams, thus illustrating atleast qualitatively the main effects of process parameters (Subramaniam et al 1997). For pharmaceutical applications biocompatible polymer micro-particles and drug-loaded poly-particles have been precipitated and protein powders with maintained biological activity have been successfully produced using the PCA technique. The application of PCA for production of superconductor and catalyst precursors and for separation purposes has also been explored. As in the case of RESS process, only a few theoretical studies have been reported mainly focusing on thermodynamics of the systems involved (DeBenedetti et al 1992). However, the application of these processes has been well documented in the past decade.

The final CSD in a batch crystallizer is determined by various kinetic processes, which include primary and secondary nucleation, crystal growth, aggregation and breakage. Because a complete theoretical model for crystallization kinetics does not exist, primary nucleation and growth rates are expressed as empirical power law equations with supersaturation as the independent variable (Mersmann 1996). The parameters of these expressions can be obtained by applying optimization techniques to fit the model predictions with experimental data. In particular, estimating the parameters of primary nucleation kinetics accurately has been a challenge, as it is impossible to detect the presence of nuclei, which are in the sub nanometer range as soon as they are formed. On the other hand, mechanistic models were developed in eighties, such as engulfment model and environments model, which were not in routine use (Pohorecky \& Baldyga 1985). Since it was observed that along with the kinetics involved, mixing played a definitive role, several authors studied the role of mixing in crystallization and precipitation reactions using different reactors in continuous as well as semi batch modes. Baldyga et al (1995), reviewed experimental data existing until then, and concluded that while it is clear that mixing controls the generation of supersaturation and thus the final CSD, it is impossible to predict intuitively the effect that the operating conditions would have on the solid characteristics. Thus it was clear that modelling approaches could provide useful tools for reactor performance prediction, design and scale-up.

5.2a CSD measurement techniques: Several CSD sensors have become available. One is a Coulter Counter, which electronically counts particles as the crystal slurry passes through an orifice. Coulter Counters have small flow orifices that are prone to clogging, especially for high 
density crystal slurries, and may require grounding of the fluid to reduce background noise (Rovang \& Randolph 1980). The forward light scattering approach is to direct a laser beam through a sample cell, and collect the light scattered through the cell. These instruments, such as the Malvern or the Microtrac particle sizers, can give useful CSD measurement for slurries with low solids density (Eek et al 1995; Eek \& Dijkstra 1995; Randolph et al 1981). Information on particle shape can be determined by examining the light intensity variations (Heffels et al 1994). This shape information can be used to correct the particle size determination using commercial laser diffraction instrumentation (Heffels et al 1996). The CSD in dense crystal slurries can be addressed by an automatic sampling and dilution unit (Jager et al 1987). However, it is challenging to collect a representative sample from an industrial-scale crystallizer and ensure that the temperature is constant enough so that the sample remains representative. The transmittance, which is the fraction of light that passes through the solution, can be measured either using a light scattering instrument or a spectrophotometer. The projected area of the crystals can be computed from the transmittance. For dense crystal slurries, the transmittance is essentially zero, and no useful information is obtained. The transmittance measurement has been used for the estimation of kinetic parameters for the crystallization of naphthalene in toluene (Witkowski et al 1990), potassium nitrate in water (Miller \& Rawlings 1994), and a photo chemical in heptane (Matthews \& Rawlings 1998). An alternative light scattering approach is based on inserting a probe directly in the crystallizer, focusing a laser beam forward through a window in the probe tip, and collecting the laser light scattered back to the probe. This approach can measure CSD information even for dense crystal slurries. One of the first commercial instruments of this type, the Par-Tec 100 analyzer, has been used to estimate kinetic parameters for the crystallization of adipic acid in water (Monnier et al 1996) as well as in feedback control (Redman et al 1997). Several publications describe applications of updated versions of the instrument, referred to as Lasentec Focused Beam Reflectance Measurement (FBRM) (Farrell \& Tsai 1995; Barrett \& Glennon 1999; Ma et al 1999; Tahti et al 1999; Togkalidou et al 2001), which are rugged enough to be implemented on industrial crystallizers. Like any laser-based method applied to crystal slurry, a transformation is required to relate the collected laser light to the CSD. The FBRM instrument measures the chord length distribution as the laser beam emitted from the sensor randomly crosses two edges of a particle, with this distance being the chord length. The random orientations of crystals can be captured as shown in figure 11.

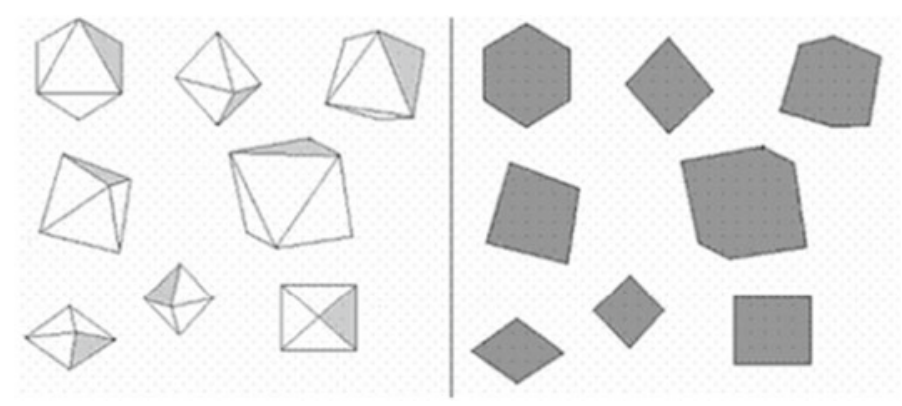

Figure 11. FBRM view: left - crystals with random orientations; right - shapes detected by FBRM with laser normal to the plane of the page (Barthe \& Rousseau 2006). 
There have been efforts to relate the chord length distribution to the particle size distribution, both by the Lasentec company and by some independent researchers (Becker 2001; Clark \& Turton 1988; Han \& Kim 1993; Liu et al 1998; Ruf et al 2000; Simmons et al 1999; Tadayyon \& Rohani1998). Chemometrics methods have been used to relate the chord length distribution to other variables, such as filtration resistance (Johnson et al 1997; Togkalidou et al 2001).

The downside of the above mentioned CSD sensors is that the distribution of crystal shape cannot be directly determined. For example, a collection of rod-like crystals are characterized mathematically by a two-dimensional distribution (one dimension being the length, and the other dimension being the breadth), but the light scattering instruments only provide one-dimensional distributions. It is impossible to uniquely determine a two-dimensional distribution from a one-dimensional distribution. The shape information is "averaged out" to obtain a onedimensional distribution.

Another instrument that has become available recently is the Lasentec Particle and Vision Measurement (PVM) system, in which pictures are taken of the crystals in solution using a probe inserted directly into the dense crystal slurry (Lasentec 1997). This video microscope can collect 10-30 pictures a second, providing two-dimensional snapshots of the crystals in real time. On-line video microscopy can image crystals as small as 5-15 microns (Lasentec 1997; Pacek et al 1994), not as small as obtained by laser scattering instruments. Also, the quality of the images limits the ability of imaging software to automatically identify individual particles and quantify the characteristics of these particles (e.g., maximum axis, minimum axis, aspect ratio). On-line video microscopy has the advantage that the crystals are directly observed, allowing shape information to be obtained. Also, the PVM in particular is a rugged instrument suitable for use in industrial applications. The main use of on-line video microscopy today is for qualitative troubleshooting, with some researchers working on how to use the images for quantitative prediction (Baier \& Widmer 2000; Braatz et al 2000). One approach is to use multiway principal component analysis, where features are tracked in the space of principal components (Bharati \& MacGregor 1998). An alternative is to take moments of the images and then to use principal components analysis to relate the image moments to characteristics of the crystals (Braatz et al 2000). Given the importance of crystal shape in applications, and that progress becomes easier as computers continue to increase in speed, it seems likely that quantitative predictions will become available.

An alternative approach to on-line video microscopy is to remove slurry from a sampling stream and flow it as a thin film over the focal region of an ordinary light microscope (Eek et al 1995; Puel et al 1997; Rawlings \& Patience 1999). A disadvantage of this approach is the requirement of having a sampling stream in which the crystals may not be representative of the actual contents in the crystallizer. A strong advantage of this approach is that the contrast between crystals and background can be made much sharper, and the number of overlapping crystals can be reduced. The images are sufficiently clean that standard image analysis algorithms can be used (Rawlings \& Patience 1999). As seen above, though in situ measurements posed the challenge of control schemes, few recent experimental techniques overcame this challenge of implementation of control schemes.

5.2b Population balance models: The objective of crystallization modelling is to provide a description of the population of crystals dispersed in the continuous phase. The mathematical framework that has been developed to describe general dispersed phase systems is known as population balance modelling. In order to model a particulate system, it is of utmost importance to decide on the stochastic or deterministic approach to be used based 
on the fluctuations in the size distributions. If the fluctuations be large and need to be modelled accurately for observables of interest, they could be described in stochastic framework. Among the various numerical techniques proposed, the broad classification can be into the following categories: (1) method of moments, in which only lower order moments of the CSD are simulated, and unknown parameters of an assumed distribution are fitted to the computed moments (Hulburt \& Katz 1964), (2) weighted residuals/orthogonal collocation methods, in which the solution is approximated as linear combinations of basis functions (Singh \& Ramkrishna 1977), (3) finite difference methods/discretized population balances (Kumar \& Ramkrishna 1996a), (4) Monte Carlo simulation, in which the histories of individual particles are tracked, each exhibiting random behaviour in accordance with a probabilistic model (Maisels et al 1999; Shah et al 1977; Song \& Qiu 1999). An exhaustive review of the available numerical methods for solving PBEs is presented by Attarakih et al $(2004,2006)$.

The advantage of the method of moments is that only a small number of ordinary differential equations needs to be solved when the moments are closed (i.e., form a finite number of equations describing the lower order moments which are not a function of the higher order moments). A weakness of the method of moments is that the moment equations are not closed for most processes, leading to an infinite number of coupled ordinary differential equations to solve. Another weakness is that, even when the moment equations are closed, the numerical errors in a fitted assumed distribution can be arbitrarily large if the assumed distribution does not accurately parameterize the true distribution. Hence a general numerical solution of the PBE cannot be developed based on the method of moments. However, the method of moments does apply to many well-mixed batch and continuous crystallizers with nucleation and growth. These assumptions can be reasonable in bench scale crystallizers such as used in teaching laboratories (Braatz et al 2002). The method of moments is also useful for testing the accuracy of more sophisticated numerical simulation codes.

Several moment based approaches came into practice in the last two decades. The quadrature method of moments (QMOM) as first introduced by McGraw (1997) to solve the PBE with pure growth is found very efficient from the accuracy and computational cost point of view. Unlike the methods such as simple finite differences or sectional methods, the QMOM has a drawback of destroying the shape of the distribution and the information about the distribution is stored only in its moments. The $\mathrm{r}^{\text {th }}$ moment is defined by integrating the population number density function with respect to certain population property (e.g., particle sizes) weighted with this property raised to its $r^{\text {th }}$ power. When $r$ equals zero, the zero moment represents the total number of particles per unit volume. On the other hand, when $r$ equals three, the third moment represents the volume fraction (volume concentration) of the particles. The QMOM tracks the population moments (e.g., the zero and third moments) rather than its size and hence it does not depend on the minimum and maximum particle sizes. A closure problem arises since the integral terms appearing in the PBE could not be written generally in terms of the moments only. This closure is due to the specific forms of the breakage and aggregation frequencies which depend in turn on the type of the physical application of the PBE. Another modified framework called Direct Quadrature Method of Moments (DQMOM) was given by Marchicio \& Fox (2005). Herein, weights and abscissas are tracked directly. Instead of solving the droplet size distribution directly, this method aims to solve some of the DSD properties, for instance the lower order moments of the distribution. The number distribution is expressed as a summation of Dirac delta functions. However the solution of the resulting linear system still becomes ill conditioned (an increase in the round off errors) and hence gets difficult to solve with sufficient accuracy.

Attarakih et al (2009) introduced a discrete framework for simulating the particulate physical systems governed by PBEs with particle splitting (breakage) and aggregation based on 
accurately (conserving (from theoretical point of view) an unlimited number of moments associated with the particle size distribution.) The basic idea is based on the concept of primary and secondary particles, where the former is responsible for distribution reconstruction while the latter is responsible for different particle interactions such as splitting and aggregation. The method is found to track accurately any set of low-order moments with the ability to reconstruct the shape of the distribution. The method is called the sectional quadrature method of moments (SQMOM) and has the advantage of being not tied to the inversion of large sized moment problems as required by the classical quadrature method of moments (QMOM). These methods become ill conditioned when a large number of moments are needed to increase their accuracy. On the contrary, the accuracy of the SQMOM increases by increasing the number of primary particles while using fixed number of secondary particles. Since the positions and local distributions for two secondary particles are found to have an analytical solution, no large moment inversion problems are anymore encountered. They concluded that generality of the SQMOM is proved by showing that all the related sectional and quadrature methods appearing in the literature till date used for solving the PBE are merely special cases.

In the application of the method of weighted residuals to the PBE, the population density is approximated by a linear combination of user-specified time-independent basis functions with time-dependent weighting factors. The basis functions are selected so that the population density can be well approximated with only a finite number of terms. The linear combination of basis functions is substituted into the PBE, and ordinary differential equations for the coefficients are derived with the intent to minimize the error (or residual) in the PBE. The system of ordinary differential equations can be solved using any standard solver (Burton et al 1951). A fast numerical algorithm results when only a small number of terms are needed in the expansion, which has been demonstrated for some crystallizers (Rawlings et al 1992; Witkowski \& Rawlings 1987). The primary weakness of the method of weighted residuals is that basis functions that work well for one type of crystallization process may not work well for another, which makes it difficult to derive a general fast algorithm for crystallization simulation using this method. This also applies to orthogonal collocation, which is essentially a class of weighted residual algorithms. Reviews of early work on the method of weighted residuals are available (Ramkrishna 1985; Rawlings et al 1993), including summaries of algorithms that combine orthogonal collocation with finite elements (Gelbard \& Seinfeld 1978). Several discretizations of the PBE have been investigated and have been applied to various particulate systems (Gelbard et al 1980; Hounslow et al 1988; Muhr et al 1996; Marchal et al 1998). This includes an application to the simulation of a crystallization process in which the crystals have two characteristic growth axes, so that changes in the crystal shape distribution are simulated (Puel et al 1997). Many of these algorithms were formulated with the intent to conserve moments of the computed population density. Different algorithms conserve different moments, and several choices of discretization points have been investigated (Kumar \& Ramkrishna 1996b). Kumar \& Ramkrishna (1996a) provide a critical review of these algorithms, including pointing out technical errors in some of the papers. Various numerical problems can occur when performing direct discretizations of the PBEs. An approach that removes these problems is to combine the discretization with the method of characteristics (Kumar \& Ramkrishna 1997; Sotowa et al 2000), which has been applied to particulate processes with pure growth, simultaneous aggregation and growth, and simultaneous nucleation and growth. High resolution finite difference schemes also avoid the numerical problems typically associated with discretizing PBEs (Ma et al 2002). The high resolution methods are able to obtain second-order accuracy without the undesirable oscillations that can occur with naive second-order methods. A high resolution method that exploits sparsity and efficiently manages memory resulted in a highly accurate dynamic simulation of the multidimensional crystal size 
distribution for a system with an extremely sharp distribution. This was a simulation of a batch crystallizer which produced prism-like crystals with two characteristic length scales and nonlinear nucleation and growth rates. Numerical analysis indicates that the method can allow a coarse time discretization, which is one of the main reasons for the short computation times. Monte Carlo methods are especially suitable for simulating stochastic PBEs, and especially for complex systems (Ramkrishna 1985).

CFD as explained in the previous section has been successfully used to model mixing controlled processes and has also been used to determine CSD of the dispersed phase. Pipino et al (1995), used a full probability density function (PDF) method and a CFD code for the flow field prediction, studied instantaneous nucleation but did not follow crystal growth. On the other hand, Wei \& Garside (1997) used a CFD code to model a precipitation reactor, but did not include a micromixing model, completely neglecting the role of mixing at a molecular level. Baldyga \& Orciuch $(1997,1999)$ discussed the effect of mixing in a tubular reactor using a presumed betaPDF coupled with CFD. Piton et al (2000) compared these results with the prediction obtained using a finite mode PDF model. They concluded that for 4-Environment Generalized Micromixing (4-EGM) model and presumed beta PDF model predict very similar results for the mean crystal size and the mean number density. The mean crystal size was found to be primarily controlled by mass transfer coefficient and the mean residence time. They also demonstrated uncertainty in prediction of mean crystal size, owing to uncertainty in exact value of mass transfer coefficient. Marchisio et al (2001), modelled barium sulfate precipitation in a semi-batch Taylor-Couette reactor with a finite mode PDF model coupled with a CFD code. They calculated the CSD in terms of first 5 moments of the CSD and validated with experimental data. This specific reactor was chosen in order to avoid breakage of crystals (unlike the case of a blade-type mixer), and provision of various fluid dynamic regimes. The CSD was measured with a Coulter LS 230 Granulometer, and solid concentration was calculated by measuring the residual reactant concentrations using conductimetric technique. They investigated the effects of various operating conditions such as inner cylinder velocity, injection velocity, initial nominal supersaturation and reactants volume ratio. They showed that the precipitation reaction is strongly influenced by the local value of supersaturation at high reactant concentrations. Further, mixing at various scales was found to be the controlling phenomenon. They showed that time dependent CFD model is the feasible way to predict the behaviour in the reactor, albeit the flow field was validated only with tracer experiments as opposed to any rigorous flow measurement techniques.

Very few work such as that by Worlitschek \& Mazzotti (2004) looked into simulating the CSD, or used a thermodynamically based solubility model. However, they did not evaluate the effect of different solubility models on the CSD prediction, leaving an open question as to which of the available solubility models is most appropriate. Wan et al (2009) developed a population balance model for predicting the dynamic evolution of crystal shape distribution which is further developed to simulate crystallization processes in which multiple crystal morphological forms co-exist and transitions between them can take place. They applied this new model to derive the optimal temperature and supersaturation profiles leading to the desired crystal shape distribution in cooling crystallization. In their work, an optimum temperature or supersaturation trajectory tracking was implemented by manipulating the coolant flow-rate in the reactor jacket. This was done by, providing a feasible closed-loop mechanism for crystal shape tailoring and control. The methodology was demonstrated by applying it to a case study of seeded cooling crystallization of potash alum.

Widenski et al (2010) compared the use of empirical, thermodynamical, and generalized thermodynamic solubility models to evaluate how these affect the resultant supersaturation profile 
and consequently the CSD, and then detailed on how to make the crystallization model more robust against solubility model error.

5.2c Integration of PBM with CFD: The modelling of well-mixed crystallizers involves the computation of the PBE together with the material balance equations for each species in solution. Numerous numerical techniques that compute the full CSD have been used to model well-mixed batch, semibatch, or continuous crystallizers (Gerstlauer et al 2002; Gunawan et al 2004; Haseltine et al 2005). To account for non-ideal mixing, the PBE has to be coupled with the transport equations of mass, momentum, and energy (Hulburt \& Katz 1964). One approach is to couple turbulent computational fluid dynamics (CFD) codes with the solution of the PBE, and most of the literature studies focus on reactive crystallization systems (Wei et al 2001; Jaworski \& Nienow 2003). Choi et al (2005) modelled the anti-solvent crystallization process in a jet Ymixer using a hybrid CFD-PBE approach but neglected the micromixing effects. Compartmental modelling, where the crystallizer is divided into a number of well-mixed compartments connected by interchanging flows, is a less computationally intensive approach. One strategy was to compartmentalize the crystallizer into regions that are, to some degree, homogeneous in properties of interest (e.g., suspension density, energy dissipation, supersaturation), as determined by CFD simulations (Kramer et al 1996). However, inaccurate compartmental modelling might oversimplify the flow field, and the spatial resolution of the supersaturation and turbulent energy dissipation distribution in the crystallizer might be lost.

Subsequently, the effects of micromixing have been included in coupled CFD-PBE computations to model turbulent precipitators (in other words-reactive crystallization), in which a variety of methods were used to approximate the probability density function (PDF), which is a statistical description of the fluctuating scalars (e.g., species concentrations) at a subgrid scale. The solution of the PBE was obtained by the method of moments, which only computes the average and aggregate properties of the crystalline phase (Kougoulos et al 2005; Baldyga \& Orcuich 2001; Marchisio et al 2001). A supercritical anti-solvent crystallization process was modelled using this strategy (Henczka et al 2005).

An alternative method used to include micromixing effects in precipitation models utilizes a multizonal approach in a Lagrangian framework, in which the precipitator is divided into a few segregated zones (e.g., feed/reactant zone, mixed/reaction zone, contact zone, bulk zone) (Baldyga \& Bourne 1999). The volume change of the zones and the material exchange between the zones are determined by the meso- and micromixing rates (Phillips et al 1999). The reduction in the computational expense by eliminating the direct linkage to CFD computations enabled the simulation of the PBE equation for the full CSD (Zauner \& Jones 2002). In some instances, additional approximations included the confinement of nucleation and crystal growth to certain zones. A variation of this approach (Kresta et al 2005) used a multiscale EulerianLagrangian frame-work to couple the zones in the bulk fluid governed by long time and length scales, and the discretized volumes of the feed plume governed by short time and length scales. Compartmental modelling, coupled to the solution of the PBE for the full particle size and shape distribution, also has been applied to cooling crystallization and polymerization processes (Alexopoulos et al 2002). Simulations of CSD took into account the spatial distribution of the solid particles of different sizes, which is important when the crystalline phase is much denser than the solution (Ma et al 2002). This was an advance over the earlier works in modelling crystallizers that assumed that the solid particles follow the liquid streamlines, which avoided the use of multiphase models. In contrast, the coupling of CFD, PBE, and multiphase models has been an ongoing effort in the modelling of bubble size distribution as a result of coalescence and 
breakup in gas-liquid processes (e.g., bioreactor). On the basis of these past efforts, it is apparent that the next step in modelling crystallizers requires a higher resolution of the flow field in order to establish a better understanding of the interactions between hydrodynamics and crystal nucleation and growth, and the impact on the CSD. Although direct numerical simulations (DNS) can resolve all flow structures of the turbulent flow and avoid the prediction of the PDF, the application of DNS in a highly turbulent flow in a full-scale reactor with chemical reactions and crystallization is still computationally intractable (Moody \& Collins 2003). Woo et al (2006) solved full CSD numerically using the high-resolution, finite-volume, semi-discrete central scheme and modelled micro-mixing by a multi-environment presumed-PDF model. They integrated this approach within commercially available CFD codes, in which the additional models are simulated within the CFD solver. The presence of solids is modelled by treating the slurry as a pseudo-homogeneous fluid with a spatial distribution of effective viscosity that depends on the local solids fraction. They investigated the effects of agitation rate, addition mode, and scaleup on the transient CSD.

Henczka et al (2005), studied numerically the supercritical anti-solvent precipitation of paracetamol with carbon-di-oxide as anti-solvent. Mass, momentum, and energy balances to take into account the heat of mixing in the studied system were solved with the commercial CFD code FLUENT. The PSD was calculated using the standard method of moments, and the interaction between mixing and precipitation was taken into account with the b-PDF approach coupled with the turbulent mixer model of Baldyga (1989). This showed the importance of including mixing effects in the model, and highlighted some limitations of the available approaches. It should be mentioned here that the micro-mixing models (which account for the interactions between turbulence and particle formation) available in the literature, are only valid for standard fluids and their extension to supercritical fluids is not straightforward. It is well known that the properties of fluids change dramatically near and above the critical point, therefore, the fluid-dynamic behaviour also changes and this directly influences mixing at all scales.

The supercritical anti-solvent (SAS) process involves the mixing of two streams in a nozzle, to get the desired thermodynamic conditions to produce supersaturation and particle formation. This process is not very different from mixing of two liquid solutions, and the resulting particle size distribution (PSD) is affected by mixing also for supercritical fluids. Sierra-Pallares et al (2012) presented a complete methodology to model precipitation systems in supercritical fluids with a dramatic change in the Schmidt number. A model to describe precipitation of b-carotene using the SAS process was developed. This model includes submodels of mixing (DQMOMIEM) and precipitation (QMOM), for analyzing phenomena associated with mixing within the precipitator. They tested different nozzles and the results obtained with the model were validated against experimental data. The predictions match well with experiments in all the cases studied. Although, due to the lack of knowledge of a simple model parameter the model cannot be used in a total predictive way, through a fitting procedure, this parameter was successfully identified. The model seems to be able to explain the experimental trend in terms of the competition between nucleation, growth, and aggregation and offers a valuable insight into the process. Precipitation by SAS is becoming more and more important due to the large amount of chemicals that can be processed with this technology as witnessed by the number of articles reported in the literature (Reverchon \& Adami 2006; Martın \& Cocero 2008).

\subsection{Multiscale multizonal models}

In order to develop models that accurately describe the real physical process, it is deemed necessary to understand the multi-scale nature of such systems ranging from fundamentals of 
crystallization phenomena at the micro-scale, to the dynamic behaviour of crystallization plants at the meso- and macro-scale. Conventionally, micro-scale represents the size scale in the vicinity of the particle physical dimension, while the meso-scale is the size scale at which fluid dynamics are considered in the crystallizer and where these dynamics affect heat transfer. The macro-scale is where lumped macro-scale variables such as the temperature and flow rate play a role. This interconnection between the different scales gives a better understanding of the design of crystallization set-up through multi-scale models.

A well-established approach to describing non-ideal mixing within process equipment is via a multizonal representation which divides the equipment volume into a network of interconnected zones where an idealized mixing pattern is assumed for each zone (Mann \& Mavros 1982; Mann et al 1987; Vlaev et al 2000). A similar method is also utilized by Vrabel et al (2000) to assess the effect of different impeller and vessel design, and of broth rheology in non-aerated and aerated stirred bioreactors. Kramer et al (1999) and Bermingham et al (2000) used hydrodynamic information to obtain a subdivision of a crystallizer into multiple compartments where phase equilibria, crystal population balance and energy balance are considered. Zauner \& Jones (2002) adopted a compartment mixing model to predict the mixing on crystal precipitation. The population balance was solved simultaneously with the mass balance using mixing data obtained by CFD calculations. However, a fundamental weakness of all multizonal models is the difficulty of characterising the mass and energy fluxes between adjacent zones. An a priori estimate of these may be obtained by means of preliminary CFD calculations. Yet, this fails to take into account the fact that the fluid properties are functions of system conditions such as temperature and PSD. Some hybrid approaches have attempted to tackle some of these issues in multizonal modelling (Bauer \& Eigenberger 1999, 2001), for a gas-liquid system. Urban \& Liberis (1999) used a hybrid multizonal/CFD modelling approach for the modelling of an industrial crystallization process. Each zone incorporates a detailed description of the crystallization phenomena in terms of a PBE. Both homogeneous and heterogeneous crystal nucleation are taken into account, the latter being a strong function of the turbulent energy dissipation rate.

A CFD model of the process is used to determine the directionality and rate of flow between adjacent zones, and the mean energy dissipation rate within each zone. The density and viscosity required by the CFD model are estimated on the basis of the CSD determined by the multizonal model. Bezzo et al (2003) modelled a dynamic bioreactor exhibiting a highly non-Newtonian behaviour. They used a hybrid approach where the multizonal model takes into account biokinetics and mass transfer, while the CFD model estimates the flow rates among zones and the effective viscosity (which is a function of the shear stress) within each zone. The mass transfer coefficient depends on the broth effective viscosity, while the parameters for the non-Newtonian description of the fluid flow are functions of the bioproduct concentration, which is estimated in the multizonal model. They developed a framework applicable to systems where the fluid dynamics operate on a much faster time-scale than other phenomena (crystallization), and can be described in terms of steady state CFD computations involving a (pseudo) homogeneous fluid, the physical properties of which are relatively weak functions of intensive properties. Abbas \& Ramagnoli (2007), developed crystallization system model, demonstrating the key steps in development of crystallization model, discussing the multi scale modelling issues. They also showed that the process can be modelled in a hybrid simulation environment, addressing the multi-scale modelling challenge. From the literature, it is shown that predictive accuracy is greatly improved by taking some account of mixing non-idealities within processing equipment. Moreover, the increase in computational complexity is relatively modest, typically being only a small multiple of the complexity of models based on idealized mixing descriptions. However, the difficulty of characterization of the flow rates between adjacent zones, and also of fluid 
mechanical quantities, such as the turbulent energy dissipation rate, that have important effects on the process behaviour within each zone at a larger scale still prevails.

\subsection{Challenges in integrating thermodynamic, kinetic and hydrodynamic models}

The challenges in crystallization process modelling are significant. The first principles approach requires a model with accurate crystallization kinetics. Firstly, uncertainties associated with their kinetics are significant. Part of the difficulty is that the kinetic parameters can be highly sensitive to small concentrations of contaminating chemicals, which can result in kinetic parameters that vary with time (Shangfeng et al 1999). Another significant source of uncertainty in industrial crystallizers is associated with mixing. Although crystallization models usually assume perfect mixing, this assumption rarely holds good for an industrial scale crystallizer. Crystallization processes are highly nonlinear, and are modelled by coupled nonlinear algebraic integro-partial differential equations. Simulating the equations which describe the shape as well as overall size, can be challenging because the CSD can be extremely sharp in practice, and can span many orders of magnitude in crystal length scale ( $\sim$ nano meter scale to millimeter range) and time scale (micro seconds to minutes).

Part of the challenge also lies with the data for validation at various levels, indicated in the modelling interplay in figure 3. Challenge includes those associated with sensor limitations. Typically, in a crystallizer, the control states include the temperature, the solute concentration, and the crystal size and shape distribution. In order to specify the nucleation and growth kinetics, the solute concentration must be measured very accurately. Because the kinetics are functions of the difference between two solute concentrations (one of these also being the saturated solute concentration), the error in the difference is much larger than the error in a single solute concentration measurement. Next challenge lies in the accurate measurement of the full CSD, though this decade offers much advanced online measurement techniques (such as FBRM). A review on

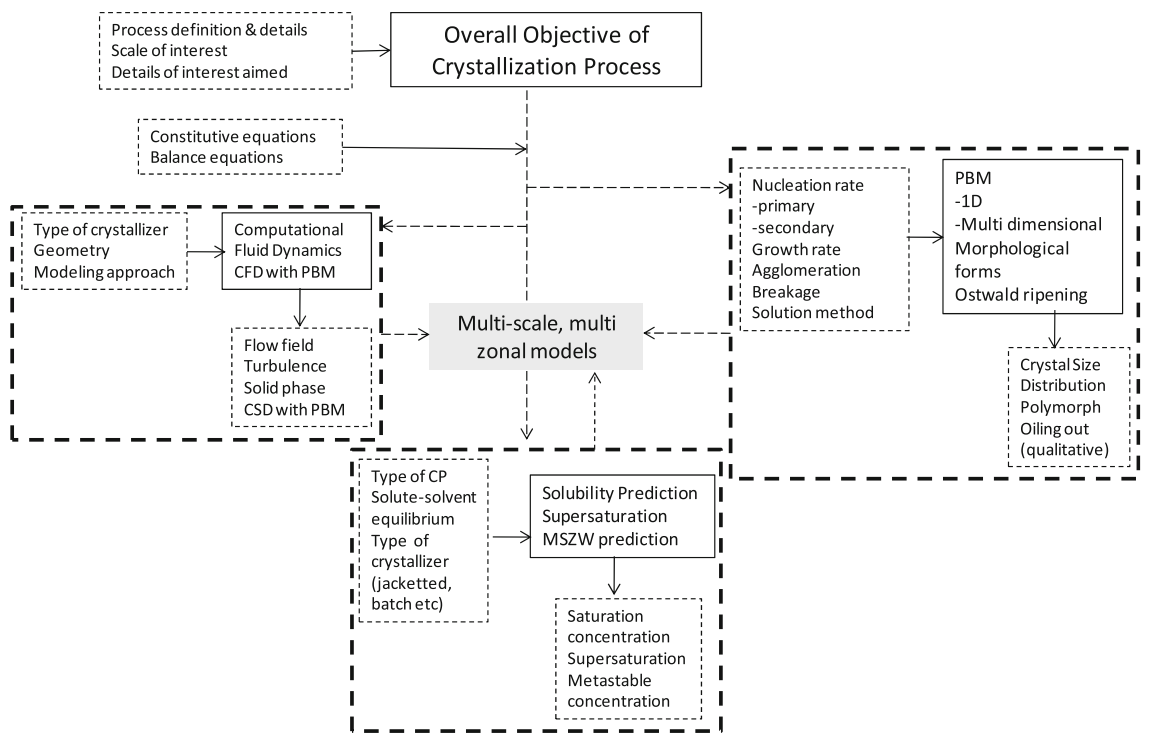

Figure 12. Overall modelling framework in a crystallization process. 
efforts to control the crystallization processes considering the above mentioned limitations was presented in a review article by Rawlings et al (1993), and Braatz (2002). However in the last decade, data analysis for in situ sensors has improved over the years due to the use of multivariate statistics for correlating spectral data and the development of better algorithms for estimating particle size distributions based on improved understanding of the operational physics involved in the process.

Mainly, the modelling framework starts with the problem definition for the crystallization process under consideration in terms of the overall objective. These extend onto constitutive equation development and framing of the balancing equations with additional process effects. In order to explain this, figure 3 could be extended to figure 12 that explains the entire modelling framework, based on the objectives to generate information on crystallization process control as well as operation based on scale-up. These could be started from any point in the entire modelling framework, and thus could lead to (one or more dimensional) generic modelling frameworks. Across the entire modelling framework, robustness and efficiency of calculations should also be tackled in order to obtain a converged and reliable solution in a reasonable time. This also indicates a further need for multizonal modelling methodology that can ultimately be applied in the context of state-of-the-art process modelling and modelling tools.

\section{Summary, conclusions and path-forward}

Crystallization is one of the key process steps for manufacturing a large number of products in fine and specialty chemicals sector (including API). Product purity and other desired product attributes (like size distribution, habit, morphology and polymorphs) are often crucial. Levels of required purity differ based on the application of the product. It is likely that the final product can have a decreased purity due to liquid trapped in agglomerates, inclusion of mother liquor in the crystal itself, specific adsorption on surfaces or formation of solid solutions (unwanted co-crystallization). These phenomena and how the process conditions can influence them, are well-described in the literature but still not predictable from one system to the other. Few trends can be pointed out as perspectives for research in the upcoming years, which are stated below.

From a thermodynamic point of view, these can be marked as follows:

- In the majority of industrial crystallization systems, typical feedback control strategies are designed to follow simple operating policies. The emergence of modern sensor technologies and advances in crystallization modelling and control has enabled more advanced control strategies to be increasingly applied. The model-based design and control approaches are based on developing a detailed model, which is used with optimization techniques to determine a dynamic supersaturation profile and/or seed recipe and addition protocol, to achieve desired product properties. The supersaturation profile is optimized indirectly by determining temperature versus time and/or anti-solvent addition rate versus time or evaporation rate trajectories. The majority of the studies until almost the early years of the past decade, focused mostly on understanding the interplay between mixing and crystallization kinetics. However, further exploitation of the interplay between these kinetic processes for the optimal operation of crystallizers remains a scarcely researched area for all types of crystallization processes.

- From the operational viewpoint, the linear cooling profile, i.e., a linear relationship between cooling temperature and time, has been identified as one of the key parameters to achieve 
the "concentration-temperature path" in solution crystallization. Whilst linear cooling profile is routinely used in laboratories, it is still impossible to be implemented in any industrial scale batch STR due to its huge volume, inherited non-uniform mixing and significant concentration/temperature gradients. Clearly, there is still a gap between the laboratory understanding and industrial-scale crystallization; the benefits being realized in laboratory STR cannot be translated into the large industrial operations.

- Prediction of solid-liquid equilibria is one of the main issues of crystallization processes. Experimental determination is not always possible, so a feasible alternative is the use of thermodynamic models which are usually used for predicting activity coefficients of liquidvapor or liquid-liquid equilibria. The main challenge in this field is to improve the accuracy of the models in order to be able to predict the solubility of molecules in complex solvents, the solubilities of solvates and also to be able to describe oiling out behaviour and to model phase equilibria.

- Although, common in the pharmaceutical industry, combined cooling and anti-solvent crystallization has not been studied in depth. Combining these could produce high supersaturations, promote the recovery of solute from the solution and thus decrease the overall energy required. But, the negative side could be the cost involved with the separation of additional solvent, and its recovery. Similarly, emerging technologies (the formation of particles using supercritical fluids, and hybrid separation processes combining crystallization with another separation technique) have been shown in literature to offer advantages, such as increased product quality, higher yield, better efficiency or being more sustainable. However, the knowledge and new hardware incur significant costs. Therefore, the economic feasibility of these technologies becomes highly important for the specific process at hand.

From a kinetic point of view, the trends can be marked as follows:

- Additional solutes/oiling out by formation of another phase, either occurring by accident or process changes usually at low concentrations, can lead to change in crystal shape or delay in crystallization. Recent development is encouraging in that simulation models are becoming available for predicting the effect of solvent type and additive concentrations on the crystal shape, especially owing to the demand of the industry. While there have been some successful works reported, more work is needed to validate the model predictions for a range of crystal systems.

- Molecular modelling could be an interesting tool for the prediction of crystal morphologies in the presence of additives or impurities. Realization of desired crystal habits would lead to reduced efforts in solid-liquid separation and to better products with respect to purity and handling. Commercial software is available to predict crystal morphology but it is not yet able to describe the complexity of molecule/solvent systems reliably. Experiments are therefore most of the time still an essential step during the development phase of a project or for trouble-shooting. Though direct prediction from models and simulation if achievable can be most advantageous, the challenges today to control product purity are mostly on the experimental level to characterize the system correctly in order to design/improve a process and to recommend the right actions for production.

- Process analytical technology (PAT) is well-known as a system for designing, analyzing, and controlling manufacturing through timely measurements (while processing) of critical quality and performance attributes of raw and in-process materials and processes, with the goal of ensuring final product quality. This also includes sensors that can measure critical process properties, such as supersaturation and desired product qualities such as size, shape 
and polymorphic form. This real-time process information can be integrated into the control algorithm to provide an optimal control strategy. Recently various tools used as PAT have been the spectroscopic methods which include IR, NIR, UV and Raman techniques. Though in practice, crystallization occurs often in multiple-component systems, these have been used in single component crystallization systems. The development of robust monitoring approaches for multi-component crystallization systems becomes mandatory if one is to use these approaches effectively.

A number of process variables affect crystallization process: supersaturation, solute concentration, temperature, mixing, cooling profile, solvent/additives and seeding. Since no complete theory is available to model nucleation/crystallization, their behaviours can only be anticipated by experimentation; process measurements are essential in understanding crystallization processes. A number of techniques have been implemented to monitor crystallization/process variables at lab scale. For example, employing optical turbidometric UV-Vis probe for the measurement of metastable zone width, X-ray diffraction (XRD) for polymorph, Fourier transform infrared spectroscopy (FTIR) for supersaturation, ultra sound spectroscopy (USS) for crystal size, focused beam reflectance measurement (FBRM) for on-line chord length of crystals and CSD, particle vision measurement (PVM) for on-line crystal shape and particle image velocimetry (PIV) for local velocity, etc. These techniques/measurements have promoted significant advances in understanding lab scale batch crystallization and have assisted in designing better crystallization processes.

However, these advances are not in coherence with an equivalent increase in our ability and understanding in scaling up stirred tank reactors - the workhorse of industrial crystallization. The scale-up of crystallization processes involves a net result of several independent, but interrelated, steps. A crystallization vessel is three-dimensional, so, as the linear dimensions increase, the capacity of the system increases as the cube of the linear dimension. With this increase in scale, other variables rise on the linear scale with different exponents, which may vary from negative to zero to three and higher. As a result, the scale-up of STRs has always been very troublesome. In addition, there is no agreement on the set of parameters to be kept constant in scale-up. This has led to some of the problems that have existed in industrial crystallization processes today: very long heating and cooling cycles due to the huge batch mass/volumes involved, large concentration and temperature gradients in plant scale crystallizers due to the non-uniform mixing and large velocity gradients making the required "concentration-temperature path" uncontrollable. Inconsistent crystal morphology, long filtration time and inconsistent product quality are some of the direct outcomes of these problems.

In addition, the techniques aforementioned provide local measurements, i.e., events at either a point or a very small area within a given crystallization vessel. The principle of such measurements is based on the assumption that the vessel is well mixed, so that local events can be used to represent the overall measurement of the whole system. At lab and small scales, such an assumption may well be valid. However, both the confidence and capability of measuring techniques decrease substantially with the increase in scale. As a result, instrumentation that has widely been used in laboratory and small scale crystallizers is seldom found in batch industrial crystallization. Even when some measuring probes are employed in large scales, the location of these probes has to be carefully selected, or else the measurements might not faithfully represent the true parameters. Also the local fluid mechanical conditions are so poor that the faces of probes get fully covered, and in some cases stuck by crystals, making measurements impossible. Furthermore, a simple task, such as implementing some of the aforementioned measurement equipment in industrial scale crystallization is still far from practical. Thus, from a hydrodynamic 
point of view, keeping in mind the importance of scale-up, design and multi-scale multizonal approach, the following can be marked as trends for research in upcoming years:

- The complexities involved in crystallization process modelling such as, non-linear dynamics, partial differential equations, significant uncertainties, unmeasured state variables, significant disturbances and sensor noise etc., pave way for challenging and interesting trends towards control of the process. The population balance methodology is well established and successful for macroscopic modelling of the dynamics of CSD.

- Since a complete CFD model cannot be expected to become available for the whole crystallization process, together with an integration of Population Balance Models as well as the CRE based models, it would be of great use to develop a modelling platform based on a compartmental model approach which on one hand communicate with CFD models and on the other hand communicate with sophisticated thermodynamic models. Some steps in that direction have been reported in the literature. However significant work is needed to develop commercially acceptable and deployable multi-scale modelling platforms.

- Although the mixing effects on precipitation are well known for liquid-based systems, such effects are not very often reported in supercritical systems. This process is not very different from mixing of two liquid solutions, and the resulting particle size distribution (PSD) is affected by mixing in a similar manner for supercritical fluids. Though, there are a number of studies supporting these evidences from the experimental point of view, little research is available in the framework of numerical modelling. As discussed in the experimental sections, extracting accurate size and shape information from in-process sensors is a very challenging theoretical problem. This calls for substantial research in this area, with proposed solutions such as merging of techniques such as digital imaging and laser backscattering information.

Validation of models is as challenging a task as building the models themselves. More validation data would result in refining models for capturing interactions between the solute and solvent molecules. Such data are expected to better our understanding of the still unclear phenomena in crystallization, and could ease the design and scale-up steps that are of great importance.

\section{References}

Abbas A and Ramagnoli J A 2007 Multiscale modeling, simulation and validation of batch cooling crystallization. Sep. Purif. Technol. 53: 153-163

Adhiyaman R and Basu S K 2006 Crystal modification of dipyridamole using different solvents and crystallization conditions. Int. J. Pharm. 321(1-2): 27-34

Adamson A W and Ghast A P 1997 Physical Chemistry of Surfaces, books.google.co.in/books?id= ENDCSAAACAAJ

Akiti O and Armenante P M 2004 Experimentally validated micro-mixing based CFD model for fed-batch stirred-tank reactors. AIChE J. 50(3): 566

Alexopoulos A H, Maggioris D and Kiparissides C 2002 CFD analysis of turbulence non-homogeneity in mixing vessels a two-compartment model. Chem. Eng. Sci. 57: 1735-1752

Al-Zoubi N and Malamataris S 2003 Effects of initial concentration and seeding procedure on crystallization of orthorhombic paracetamol from ethanolic solution. Int. J. Pharmaceutics. 260: 123-135

Attarakih M M, Bart H J and Faqir N M 2004 Numerical solution of the spatially distributed population balance equation describing the hydrodynamics of interacting liquid-liquid dispersions. Chem. Eng. Sci. 59(12): 2567-2592 
Attarakih M M, Bart H J and Faqir N M 2006 Numerical solution of the bivariate population balance equation for the interacting hydrodynamics and mass transfer in liquid-liquid extraction columns. Chem. Eng. Sci. 61(1): 113-123

Attarakih M M, Drumm C, Bart H J and Faqir N M 2009 Solution of the population balance equation using the sectional quadrature method of moments (SQMOM). Chem. Eng. Sci. 64: 742-752

Avrami M 1939 Kinetics of phase change. I. General theory. J. Chem. Phys. 7(12): 1103-1112

Baier F O and Widmer F 2000 Measurement of the bubble size distribution in a gas-liquid-contactor using the PVM and image analysis. Technical report, Institute of Process Engineering, Swiss Federal Institute (ETH), Zurich, Switzerland

Baldyga J 1989 Turbulent mixer model with application to homogeneous, instantaneous chemical reactions. Chem. Eng. Sci. 44: 1175-1182

Baldyga J and Bourne J R 1999 Turbulent mixing and chemical reactions. Wiley: Chichester, UK

Baldyga J and Orciuch W 1997 Closure problem for precipitation. Chem. Eng. Res. Des. 75A: 160

Baldyga J and Orciuch W 1999 Closure method for turbulence in inhomogeneous turbulence. In: Proc. of 14th Symp. on Ind. Crystallization, 1069

Baldyga J and Orcuich W 2001 Barium sulphate precipitation in a pipe: an experimental study and CFD modeling. Chem. Eng. Sci. 56: 2435-2444

Baldyga J, Podgorska W and Pohorecky R 1995 Mixing precipitation model with application to double feed semibatch precipitation. Chem. Eng. Sci. 50: 1281

Bakker A and van den Akker H E A 1994 A computational model for the gas-liquid flow in stirred reactors. Trans. Inst. Chem. Eng. 72: 594-606

Barrett P and Glennon B 1999 In-line FBRM monitoring of particle size in dilute agitated suspensions. Part. Syst. Charact. 16(5): 207-211

Barret P and Glennon B 2002 Characterizing the metastable zone width and solubility curve using LASENTEC FBRM and PVM. Trans. I. Chem. E. 80 (Part A): 945-952

Barrett P, Smith B, Worlitschek J, Bracken V, Sullivan B and Grady D 2005 A review of the use of process analytical technology for the understanding and optimization of production batch crystallization processes. Org. Proces. Res. \& Develop. 9: 348-355

Barthe S and Rousseau R W 2006 Utilization of FBRM in the control of CSD in a batch cooled crystallizer. Chem. Eng. Tech. 29(2): 206-211

Bauer M and Eigenberger G 2001 Multiscale modelling of hydrodynamics, mass transfer and reaction in bubble column reactors. Chem. Eng. Sci. 56: 1067-1074

Becker R 2001 Attributes and limitations of FBRM and PVM for scale-up and troubleshooting of crystallization processes. In: 11th Annual Meeting of the Association for Crystallization Technology, Northbrook, Illinois

Bermingham S K, Neumann A M, Kramer H J M, Verheijen P J T, van Rosemalen G M and Grievink J 2000 AIChE Symp. Ser. 96: 250

Bezzo F, Macchietto S and Pantelides C C 2003 General hybrid multizonal/CFD approach for bioreactor modelling. AIChE J. 49: 2133-2148

Bharati M H and MacGregor J F 1998 Multivariate image analysis for real-time process monitoring and control. Ind. Eng. Chem. Res. 37(12): 4715-4724

Blagden N, Song M, Davey R J, Seton L and Seaton C C 2005 Ordered aggregation of benzamide crystals induced using a "motif copper" additive. Cryst. Growth \& Des. 5(2): 467-471

Bladgen N, deMatas M, Gavan P T and York P 2007 Crystal engineering of active pharmaceutical ingredients to improve solubility and dissolution rates. Adv. Drug. Deliv. Rev. 59(7): 617-630

Bonnett P E, Carpenter K J, Dawson S and Davey R J 2003 Solution crystallization via a submerged liquid-liquid phase boundary: Oiling out. Chem. Comm. 6: 698-699

Braatz R D 2002 Advanced control of crystallization processes. Annu. Rev. Control. 26: 87-99

Braatz R D, Ma D L, Togkalidou T, Fujiwara M, Patel S D and Tafti D K 2000 Modeling and control of multidimensional crystallization. AIChE Annual Meeting, Los Angeles, California, Paper $253 \mathrm{~h}$

Braatz R D, Fujiwara M, Ma D L, Togkalidou T and Tafti D K 2002 Int. J. Mod. Phys. B 16: 346-353

Brittain H G 1999 Polymorphism in Pharmaceutical Solids, Ronier Lenin Hernandez Rueda 
Bungert B, Sadowski G and Arlt W 1998 Separations and material processing in solutions with dense gases. Ind. Eng. Chem. Res. 37: 3208-3220

Burton W K, Cabrera N and Frank F C 1951 The growth of crystals. Philos. Trans. R. Soc. 243: 299358

Cacciuto A, Auer S and Frenkel D 2004 Onset of heterogeneous crystal nucleation in colloidal suspensions. Nature 428: 404-406

Chen C M and Higg P G 1998 J. Chem. Phys. 108: 4305

Choi Y J, Chung S T, Oh M and Kim H S 2005 Cryst. Growth Des. 5: 959-968

Clark N N and Turton R 1988 Chord length distributions related to bubble size distributions in multiphase flows. Int. J. Multi-phase Flow 14(4): 413-424

Codan L, Babler M U and Mazzotti M 2011 Design of crystallization processes for the resolution of conglomerate-forming chiral compounds exhibiting oiling out. In: 18th International Symposium on Industrial Crystallization, Zurich, Switzerland, September 13-16th

Davey R J, Allen K and Bladgen N 2002 Crystal engineering-nucleation, the key step. J. Cryst. Eng. Commun. 4(47): 257-264

David R, Villermaux J, Marchal P and Klein J P 1991 Crystallization and precipitation engineering - IV. Kinetic model of adipic acid crystallization. Chem. Eng. Sci. 46: 1129

DeBenedetti P G, Tom J W, Kwauk X and Yeo S D 1992 Rapid expansion of supercritical solutions (RESS): Fundamentals and Applications. Fluid Phase Equilibria. 82: 311-321

Deneau E and Steele G 2005 An in-line study of oiling out and crystallization. Org. Proc. Res. Dev. 9: 943-950

Diepen PJ 1998 Cooling crystallization of organic compounds. PhD-Thesis, Delft University of Technology, Delft

Dimonte J E, Szutowski H and Niederberger H 2000 Salt Crystallization Systems: Design Features \& Operating Advantages. In: Proc 8th World Salt Symposium, vol. 1, Elsevier, The Hague

Dirksen J A and Ring T A 1991 Fundamentals on crystallization: Effects on PSDs and morphology. Chem. Eng. Sci. 46(10): 2389-2427

Doye J K and Frenkel D 1999 Kinetic Monte Carlo simulations of the growth of polymer crystals. J. Chem. Phys. 110: 2692

Draucker L C, Janakat M, Lazzaroni M J, Bush D, Eckert C A, Frank T C and Olson J D 2007 Experimental determination and model prediction of solid solubility of multifunctional compounds in pure and mixed nonelectrolyte solvents. Ind. Eng. Chem. Res. 46: 2198-2204

Dunitz JD 2003 Crystal and co-crystal- A second opinion. Cryst. Engng. Comm. 5: 506

Eek RA and Dijkstra S 1995 Design and experimental evaluation of a state estimator for a crystallization process. Ind. Eng. Chem. Res. 34: 567-574

Eek R A, Dijkstra S and van Rosmalen G M 1995 Dynamic modeling of suspension crystallizers, using experimental data. AIChE J. 41(3): 571-584

Evans U R 1945 Trans. Faraday Soc. 41: 365

Farrell R J and Tsai Y C 1995 Nonlinear controller for batch crystallization: development and experimental demonstration. AIChE J. 41(10): 2318-2321

Feng L and Berglund K 2002 ATR-FTIR for determining optimal cooling curves for batch crystallization of succinic acid. Cryst. Growth Des. 2(5): 449-452

Févotte G and Klein J P 1994 Application of on-line calorimetry to the advanced control of batch crystallizers. Chem. Engng. Sci. 49: 1323-1336

Févotte G and Klein J P 1996 A new policy for the estimation of the course of supersaturation in batch crystallization. Can. J. Chem. Engng. 74: 372-384

Foddi O, Abbas A, Grosso M and Romagnoli J A 2007 Modeling, validation and optimization of antisolvent crystallization: A new approach in predicting solubility using a thermodynamic model. A.I.Ch.E. Conf.

Frawley P J, Mitchell N A, O'Ciardha C T and Hutton K W 2012 The effects of supersaturation, temperature, agitation and seed surface area on the secondary nucleation of paracetamol in ethanol solutions. Chem. Eng. Sci. 75: 183-197 
Fujiwara M, Chow P S, Ma D L and Braatz R D 2001 Paracetamol crystallization using laser back-scattering and ATR-FTIR spectroscopy: metastability, agglomeration and control. Cryst. Growth \& Des. 2(5): 363370

Garcia E, Veesler S, Biostelle R and Hoff C 1999 Crystallization and dissolution of pharmaceutical compounds: An experimental approach. J. Cryst. Growth 198-199(2): 1360-1364

Gelbard F and Seinfeld J H 1978 Numerical solution of the dynamic equation for particulate systems. J. Comput. Phys. 28: 357-375

Gelbard F, Tambour Y and Seinfeld J H 1980 Sectional representations for simulating aerosol dynamics. J. Colloid Interface Sci. 76(2): 541-556

Gerstlauer A, Motz S, Mitrovic A and Gilles E D 2002 Development, analysis and validation of population models for continuous and batch crystallizers. Chem. Eng. Sci. 57: 4311-4327

Gosman A D, Lekakou C, Politis S, Issa R I and Looney M K 1992 Multi-dimensional modeling of turbulent two-phase flows in stirred reactors. AIChE J. 38(12): 1947

Guha D, Ramchandran P A and Duduckovic M 2007 Flow field of suspended solids in a stirred tank rector by Lagrangian tracking. Chem. Eng. Sci. 62: 6143

Guiraud P, Costes J and Bertrand J 1997 Local measurements of fluid and particle velocities in stirred suspension. Chem. Eng. J. 68: 75-86

Gunawan R, Fusman I and Braatz R D 2004 High resolution algorithms for multidimensional population balance equations. AIChE J. 50: 2738-2749

Han J H and Kim S D 1993 Bubble chord length distribution in three-phase fluidized beds. Chem. Eng. Sci. 48(6): 1033-1039

Haseltine E L, Patience D B and Rawlings J B 2005 On the stochastic simulation of particulate systems. Chem. Eng. Sci. 60: 2627-2641

Heffels C M G, Heitzmann D, Hirleman E and Scarlett B 1994 The use of azimuthal intensity variations in diffraction patterns for particle shape characterization. Part. Part. Syst. Charact. 11: 194199

Heffels C M G, Verheijen P J T, Heitzmann D and Scarlett B 1996 Correction of the effect of particle shape on the size distribution measured with a laser diffraction experiment. Part. Part. Syst. Charact. 13: 271-279

Helt J E and Larson M A 1977 Effects of temperature on the crystallization of potassium nitrate by direct measurement of supersaturation. AIChE J. 23(6): 822-830

Henczka M, Baldyga J and Shekunov B Y 2005 Particle formation by turbulent mixing with supercritical anti-solvent. Chem. Eng. Sci. 60: 2193-2201

Hojjati H and Rohani S 2006 Measurement and prediction of solubility of paracetamol in waterisopropanol solution: Part 1 - measurement and data analysis. Org. Proces. Res \& Develop. 10: 11011117

Hollander E D, Derksen J J, Bruinsma O S L, van der Akker H E A and van Rosmalen G M 2001 A numerical study on the coupling of hydrodynamics and orthokinetic agglomeration. Chem. Eng. Sci. 56: 2531

Hounslow M J, Ryall R L and Marshall V R 1988 A discretized population balance for nucleation, growth, and aggregation. AIChE J. 34(11): 1821-1832

Hu Q, Rohani S, Wang D X and Jutan A 2005 Optimal control of a batch cooling seeded crystallizer. Powder Technol. 156(2-3): 170-176

Hulburt H M and Katz S 1964 Some problems in particle technology. Chem. Eng. Sci. 19: 555574

Jager J, de Wolf S, Klapwijk W and de Jong E J 1987 A new design for on-line product-slurry measurements, Industrial Crystallization 87, Chemical Engineering Progress Symposium Series, pages 415-418, Bechyne, Czechoslovakia: Elsevier Science

Jans B J, Bischoff R, Fischer O and Wynn N 1996 Heat Pump Crystallizer. In: Proc. 13th Symp. on Industrial Crystallization, Toulouse, 539

Jansens P J and Matsuoka M 2000 Encyclopedia of Separation Science, Vol. 3, Chap. II, Melt Crystallization. London: Academic Press 
Jaworski Z and Nienow A W 2003 CFD modelling of continuous precipitation of barium sulphate in a stirred tank. Chem. Eng. J. 91: 167-174

Johnson B K, Szeto C, Davidson O and Andrews A 1997 Optimization of pharmaceutical batch crystallization for filtration and scale-up, In AIChE J., Paper 16a

Jones A G 2002 Crystallization process systems. Butterworth-Heinemann

Joshi J B, Nere N K, Rane C V, Murthy B N, Mathpati C S, Patwardhan A W and Ranade V V 2011 CFD simulation of stirred tanks: Comparison of turbulence models (Part II: Axial Flow Impellers, Multiple Impellers and Multiphase Dispersions). The Canad. J. Chem. Eng. 89: 754-816

Jung J and Perrut M 2001 Particle using supercritical fluids: Literature and patent survey. J. Supercritical Fluids 20: 179-219

Karpinski P H 2006 Polymorphism of active pharmaceutical ingredients. Chem. Eng. Technol. 29(2): 233237

Khopkar A R, Kasat G R, Pandit A B and Ranade V V 2006 Computational fluid dynamics simulation of the solid suspension in stirred slurry reactor. Ind. Chem. Res. Des. 45: 4416

Kiesow K, Tumakaka F and Sadowski G 2008 Experimental investigation and prediction of oiling out during crystallization process. J. Cryst. Growth. 310: 4163-4168

Knox M, Trifkovic M and Rohani S 2009 Combining anti-solvent and cooling crystallization: Effect of solvent composition on yield and meta stable zone width. Chem. Eng. Sci. 64: 3555-3563

Koenig H A 1998 Modern computational methods. Philadelphia, PA: Taylor \& Francis

Kougoulos E, Jones A G and Wood-Kaczmar M 2005 Chem. Eng. Res. Des. 83: 30-39

Kramer H J M and Jansens P J 2003 Tools for design and control of industrial crystallizers-state of the art \& future needs. Chem. Eng. Technol. 26(3): 247-255

Kramer H J M, Dijkstra J W, Neumann A M, Meadhra R O and van Rosmalen G M 1996 Modelling of industrial crystallizers, a compartmental approach using a dynamic flow-sheeting tool. J. Cryst. Growth 166: $1084-1088$

Kramer H J M, Bermingham S K and van Rosmalen G M 1999 Design of industrial crystallisers for a given product quality. J. Cryst. Growth 199: 729-737

Kresta S, Anthieren G and Parsiegla K 2005 Chem. Eng. Sci. 60: 2135-2153

Kubota N 2010 A unified interpretation of metastable zone widths and induction times measured for seeded solutions. J. Cryst. Growth 312: 548-554

Kubota N, Doki N, Yokots M and Jagdeesh D 2001 Seeding policy in cooling crystallization. Pow. Tech. 121: $31-38$

Kumar S and Ramkrishna D 1996a On the solution of population balance equations by discretization-I. A fixed pivot technique. Chem. Eng. Sci. 51(8): 1311-1332

Kumar S and Ramkrishna D 1996b On the solution of population balance equations by discretization-II. A moving pivot technique. Chem. Eng. Sci. 51(8): 1333-1342

Kumar S and Ramkrishna D 1997 On the solution of population balance equations by discretization-III. Simultaneous nucleation, growth and aggregation. Chem. Eng. Sci. 52: 4659-4679

Kwang-Joo J and Mersmann A 2001 Estimation of meta stable zone width in different nucleation processes. Chem. Eng. Sci., Industrial Crystallization 56(7): 2315-2324

Lafferrere L, Hoff C and Veesler S 2004 In-situ monitoring of the impact of liquid-liquid phase separation on drug crystallization by seeding. Cryst. Growth Des. 4(6): 1175-1180

Lasentec, Lasentec 1997, Brochure

Liu W, Clark N N and Karamavruc A I 1998 Relationship between bubble size distributions and chordlength distribution in heterogeneously bubbling systems. Chem. Eng. Sci. 53(6): 1267-1276

Ljungqvist M and Rasmuson A 2001 Numerical simulation of the two-phase flow in an axially stirred reactor. Trans. IChemE 79(Part A): 533

Llinas A and Goodman J M 2008 Polymorph control: past, present and future. Drug Disc. Today 13(5/6): 198-210

Ma D L, Togkalidou T and Braatz R D 1999 Multidimensional crystal growth from solution, In: AIChE Annual Meeting, Paper 03A02

Ma D L, Braatz R D and Tafti D K 2002 Int. J. Mod. Phys. B. 16: 383-390 
Maisels A, Kruis F E and Fissan H 1999 Direct Monte Carlo simulations of coagulation and aggregation. J. Aerosol Science 30: S417-S418

Mandelken L 2001 Crystallization of Polymers, 2nd ed. Cambridge: Cambridge University Press

Mann R and Mavros P 1982 Analysis of unsteady tracer dispersion and mixing in a stirred vessel using interconnected networks of ideal flow zones. In: Papers presented at the 4th European conf. on mixing (pp. 35-47), Noordwijkerhout, The Netherlands, April 1982. Cranfleld, Bedford, UK: BHRA Fluid Engineering

Mann R, Knysh P, Rasekoala E A and Didari M 1987 Mixing in a closed stirred vessel: Use of networks of zones to interpret mixing in a closed stirred vessel, In: Fluid mixing: Vol. III. International Chemical Engineering Symposium Series (No. 108, pp. 49-60)

Marchicio D L and Fox R O 2005 Solution of the population balance equation using the direct quadrature method of moments. J. Aerosol Science 36: 43-73

Marchal P, David R, Klein J P and Villermaux J 1998 Crystallization and precipitation engineering. I: An efficient method for solving population balance in crystallization with agglomeration. Chem. Eng. Sci. 43: 59-67

Marchisio D, Barressi A A and Fox R O 2001 Simulation of turbulent precipitation in a semi-batch TaylorCouette reactor using CFD. AIChE J. 47(4): 664

Marqusee J A and Ross J 1983 Kinetics of phase transitions: Theory of Ostwald ripening. J. Chem. Phys. 79: $373-378$

Martın A and Cocero M 2008 Micronization processes with supercritical fluids: Fundamentals and mechanisms. Adv. Drug Delivery Rev. 60: 339-350

Matthews H B and Rawlings J B 1998 Batch crystallization of a photochemical: Modeling, control and filtration. AIChE J. 44(5): 1119-1127

McCoy B and Madras G 2008 Cluster kinetics of phase transitions: Applications to innovative technologies. Chem. Eng. Commun. 196: 204-233

McGraw R 1997 Description of aerosol dynamics by the quadrature method of moments. Aerosol Sci. Technol. 27: 255-265

McKeown R R, Wertman J T and Dell'Orco C 2011 Crystallization Design and Scale-up, Chemical Engineering in the Pharmaceutical Industry, John Wiley \& Sons, Inc., Publication, 213-248

Meares P 1965 Polymers: Structure and properties. New York: Van Nostrand

Mersmann A 1996 Supersaturation and nucleation. Chem. Eng. Res. Des. 74: 812

Mersmann A and Bartosch K 1998 How to predict metastable zone width. J. Cryst. Growth 183: 240-250

Micale G, Montante G, Grisafi F, Brucato A and Godfrey J 2000 CFD simulation of particle distribution in stirred reactors. Trans. IChemE 78(Part A): 435

Micale G, Grisafi F, Rizzuti L and Brucato A 2004 CFD simulation of particle suspension height in stirred vessels. Chem. Eng. Res. Des. 82: 1204

Miller S M and Rawlings J B 1994 Model identification and control strategy for batch cooling crystallizers. AIChE J. 40(8): 1312-1327

Mirmehrabi M, Rohani S and Perry L 2006 Thermodynamic modeling of activity coefficient and prediction of solubility: Part 2. Semipredictive or semi empirical models. J. Pharm. Sci. 95(4): 798-809

Monnier O, Klein J P, Hoff C and Ratsimba B 1996 Particle size determination by laser reflection: methodology and problems. Part. Part. Syst. Charact. 13: 10-17

Montante G and Magelli F 2005 Modelling of solids distribution in stirred tanks: Analysis of simulation strategies and comparison with experimental data. Int. J. Comput. Fluid Dyn. 19: 253

Moody E G and Collins L R 2003 Effect of mixing on the nucleation and growth of Titania particles. Aerosol Sci. Technol. 37: 403

Muhr H, David R, Villermaux J and Jezequel P H 1996 Crystallization and precipitation engineering - VI. Solving population balance in the case of the precipitation of silver bromide crystals with high primary nucleation rates by using the first order upwind differentiation. Chem. Eng. Sci. 51(2): 309-319

Mullin J W 2001 Crystallization-Fourth Edition. Butterworth-Heinemann

Mullin J W and Leci C J 1972 Desupersaturation of seeded citric acid solutions in a stirred vessel. AIChE Symp. Ser. 68(121): 8-20 
Myerson A 2002 Handbook of industrial crystallization. Butterworth-Heinemann

Nagy Z K, Fujiwara M, Woo X Y and Braatz R D 2008 Determination of the kinetic parameters for the crystallization of paracetamol from water using metastable zone width experiments. Ind. Eng. Chem. Res. 47: 1245-1252

Nienow A W 1985 In: Mixing in the process industries, N Harnby et al (eds), Butter-worth, London

Nowee S M, Abbas A and Romagnoli J A 2008 Model-based optimal strategies for controlling particle size in anti-solvent crystallization operations. Cryst. Growth Des. 8(8): 2698-2706

Nyvlt J, Sohnel O, Matachova M and Broul M 1985 The kinetics of industrial crystallization. Elsevier

Ohtaki H 1998 Crystallization processes. Wiley

Pacek A W, Moore I P T, Nienow A W and Calabrese R V 1994 Video technique for measuring dynamics of liquid-liquid dispersion during phase inversion. AIChE J. 40: 1940-1949

Paul E L, Atiemo-Obeng V A and Kresta S 2004 Handbook of industrial mixing science and practice. John-Wiley \& Sons Publication

Phillips R, Rohani S and Baldyga J 1999 Micromixing in a single-feed semi-batch precipitation process. AIChE J. 45: 82-92

Pinelli D, Nocentini M and Magelli F 2001 Solids distribution in stirred slurry reactors: influence of some mixer configurations and limits to the applicability of a simple model for predictions. Chem. Eng. Commun. 188: 91-107

Pipino M, Barresi A A and Fox R O 1995 A PDF approach to the description of homogeneous nucleation. In: Proc. of 4th Int. Conf. on Multiphase Flow in Ind. Plants, Ancona, Italy, page 245

Piton D, Fox R O and Marcant B 2000 Simulation of fine particle formation by precipitation using computational fluid dynamics. Can. J. Chem. Engng. 78: 983

Pohorecky R and Baldyga J 1985 The effect of micromixing on the course of precipitation in an unpremixed feed continuous crystallizer. In: Proc. of 4th Eur. Conf. on Mixing (BHRA), Wurzburg, West Germany, page 105

Puel F, Marchal P and Klein J 1997 Habit transient analysis in industrial crystallization using two dimensional crystal size technique. Chem. Eng. Res. Dev. 75: 193-205

Purohit R and Venugopalan P 2009 Polymorphism: An overview. Resonance, pp. 882-893

Ramkrishna D 1985 The status of population balances. Rev. Chem. Eng. 3(1): 49-95

Randolph A D, White E T and Low C D 1981 On-line measurement of fine-crystal response to crystallizer disturbances. Ind. Eng. Chem. Proc. Des. Dev. 20: 496-503

Rawlings J B and Patience D B 1999 On-line monitoring and control of crystal size and shape. Ann. Meeting of the International Fine Particle Research Institute, Somerset, New Jersey

Rawlings J B, Witkowski W R and Eaton J W 1992 Modelling and control of crystallizers. Powder Technol. 69: 3-9

Rawlings J B, Miller S M and Witkowski W R 1993 Model identification and control of solution crystallization processes: A review. Ind. Eng. Chem. Res. 32: 1275-1296

Redman T P and Rohani S 1994 On-line determination of supersaturation of a KCL-NaCL aqueous solution based on density measurement. Can. J. Chem. Engng. 72(1): 64-71

Redman T, Rohani S and Strathdee G 1997 Control of the crystal mean size in a pilot plant potash crystallizer. Chem. Eng. Res. Dev. 75: 183-192

Reverchon E and Adami R 2006 Nanomaterials and supercritical fluids. J. Supercrit Fluids. 37: $1-22$

Rewatkar V B and Joshi J B 1991 Critical impeller speed for solid suspension in mechanically agitated three-phase reactors. 2. Mathematical model. Ind. Eng. Chem. Res. 30: 1784

Rohani S, Horne S and Murthy K 2005 Control of product quality in batch crystallization of pharmaceuticals and fine chemicals. Part 2. External Control. Org. Proc. Res. \& Dev. 9: 873-883

Rovang R D and Randolph A D 1980 On-line particle size analysis in the fines loop of a KCl crystallizer. Design, Control, and Analysis of Crystallization Processes, vol. 76, pages 18-26. AIChE, New York. AIChE Symp. Ser.

Ruf A, Worlitschek J and Mazzotti M 2000 Modeling and experimental analysis of PSD measurements through FBRM. Part. \& Part. Syst. Charac. 17(4): 167-179 
Sardeshpande M V, Sagi A R, Juvekar A V and Ranade V V 2009 Solid suspension and liquid phase mixing in solid-liquid suspensions. Ind. Eng. Chem. Res. 48: 9713-9722

Sardeshpande M V, Juvekar A V and Ranade V V 2011 Solid suspensions in stirred tanks: UVP measurements and CFD simulations. Canad. J. Chem. Eng. 89: 1112-1121

Sbrizzai F, Lavezzo V, Verzicco R, Campolo M and Soldati A 2006 Direct numerical simulation of turbulent particle dispersion in an unbaffled stirred-tank reactor. Chem. Eng. Sci. 61: 2843-2851

Schmidt B, Patel J, Ricard F X, Brechtelsbauer C M and Lewis N 2004 Application of process modelling tools in the scale-up of pharmaceutical. Org. Process Res. Dev. 8: 998-1008

Scholl J, Vicum L, Muller M and Mazzotti M 2006 Precipitation of L-glutamic acid: Determination of nucleation kinetics. Chem. Eng. Technol. 29(2): 257-264

Sha Z, Louhi-Kultanen M, Oinas P and Palosaari S 1999 CFD simulation of size dependent classification in an imperfectly mixed suspension crystallizer. In: Proc. of the ldth Int. Syrup. on Industrial Crystallization, Cambridge, UK

Sha Z, Palosaari S, Oinas P and Ogawa K 2001 CFD simulation of solid suspension in a stirred tank. J. Chem. Eng. Jpn. 34: 621

Shah B H, Ramkrishna D and Borwanker J D 1977 Simulation of particulate systems using the concept of the interval of quiescence. AIChE J. 23: 897-904

Shangfeng Y, Genbo S, Zhengdong L and Rihong J 1999 Rapid growth of KH2 PO4 crystals in aqueous solutions with additives. J. Cryst. Growth 197: 383-387

Shekunov B Y and Grant D J W 1997 In Situ optical interferometric studies of the growth and dissolution behaviour of paracetamol (Acetaminophen): 1. Growth kinetics. J. Phys. Chem. B. 101: 3973-3979

Sierra-Pallares J, Marchisio D L, Parra-Santos M T, García-Serna J, Castro F and Cocero M J 2012 A computational fluid dynamics study of supercritical anti-solvent precipitation: Mixing effects on particle size. AIChE J. 58: 385-398. doi:10.1002/aic.12594

Simmons M J H, Langston P A and Burbidge A S 1999 Particle and droplet size analysis from chord distributions. Pow. Tech. 102(1): 75-83

Singh P N and Ramkrishna D 1977 Solution of population balance equations by MWR. Comput. Chem. Eng. 1: 23-31

Sohnel O and Garside J 1992 Precipitation: Basic principles and industrial applications. ButterworthHeinemann

Song M and Qiu XJ 1999 Alternative to the concept of the interval of quiescence (IQ) in the Monte Carlo simulation of population balances. Chem. Eng. Sci. 54: 5711-5715

Sotowa K, Naito K, Kano M, Hasebe S and Hashimoto I 2000 Application of the method of characteristics to crystallizer simulation and comparison with finite difference for controller performance evaluation. J. Process Control 10: 203-208

Subramaniam B, Rajewski R A and Snavley K 1997 Pharmaceutical processing with supercritical carbondi-oxide. J. Pharm. Sci. 86: 885-890

Tadayyon A and Rohani S 1998 Determination of particle size distribution by Par-TecR 100: Modeling and experimental results. Part. Part. Syst. Charact. 15(3): 127-135

Tahti T, Louhi-Kultanen M and Palosaari S 1999 On-line measurement of crystal size distribution during batch crystallization. In: Proceedings of 14th International Symposium on Industrial Crystallization, Cambridge, UK

Tavare N S 1987 Simulation of Ostwald ripening in a batch crystallizer. AIChE J. 33(1): 152

ter Horst J H, Kramer H J M, van Rosmalen G M and Jansens P J 2002 Molecular modeling of the crystallization of polymorphs. Part I: The morphology of HMX polymorphs. J. Cryst. Growth 237-239: 2215-2220

Togkalidou T, Fujiwara M, Patel S and Braatz R D 2001 Solute concentration prediction using chemometrics and ATR-FTIR spectroscopy. J. Cryst. Growth 231: 534-543

Tom J W and Debenedetti P G 1991 Particle formation with supercritical fluids: A review. J. Aerosol Sci. 22: $555-584$

Tung H H, Paul E L, Midler M and Mc Cauley J A 2009 Crystallization of organic compounds. An industrial perspective. John Wiley \& Sons 


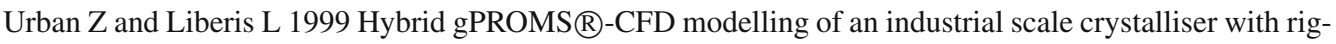
orous crystal nucleation and growth kinetics and a full population balance. In: Proceedings: Chemputers 1999 conf., Düsseldorf, Germany

Variankaval N, Kote A S and Doherty M F 2008 From form to function: Crystallization of active pharmaceutical ingredients; 2008. AIChE J. 54(7): 1682-1688

Veesler S, Lafferrere L, Garcia E and Hoff C 2003 Org. Process Res. Dev. 7: 983

Vlaev D, Mann R, Lossev V, Vlaev S V, Zahradnik J and Seichter P 2000 Macro-mixing and Streptomyces Fradiae. Modelling oxygen and nutrient segregation in an industrial bioreactor. Chem. Eng. Res. Des. 78: $354-362$

Vrabel P, van der Lans R G J M, Luyben K C A M, Boon L and Nienow A W 2000 Mixing in largescale vessels stirred with multiple radial or radial and axial up-pumping impellers: Modelling and measurements. Chem. Eng. Sci. 55: 5881-5896

Wan J, Wang X Z and Ma C Y 2009 Particle shape manipulation and optimization in cooling crystallization involving multiple crystal morphological forms. AIChE J. 55(8): 2049-2061

Wei H and Garside J 1997 Application of CFD modeling to precipitation systems. Chem. Eng. Res. Des. 75a: 219

Wei H Y, Zhou W and Garside J 2001 Computational fluid dynamics modeling of the precipitation process in a semibatch crystallizer. Ind. Eng. Chem. Res. 40: 5255-5261

Widenski D J, Abbas A and Romagnoli J A 2010 Comparison of different solubility equations for modeling in cooling crystallization. Chem. Eng. Process. 49: 1284-1297

Winn D and Doherty M F 2000 Modeling crystal shapes of organic materials grown from solution. AIChE J. 46: $1348-1367$

Witkowski W R, Miller S M and Rawlings J B 1990 Light-scattering measurements to estimate kinetic parameters for crystallization. ACS Symp. Ser. 438: 102-114

Witkowski W R and Rawlings J B 1987 Modelling and control of crystallizers. In: Proc. of the American Control Conf. Piscataway, New Jersey: IEEE Press, pp. 1400-1405

Woo X Y, Tan R B H, Chow P S and Braatz R D 2006 Simulation of mixing effects in anti-solvent crystallization using a coupled CFD-PDF-PBE approach. Cryst. Growth Des. 6(6): 1291-1303

Worlitschek J 2003 Monitoring, modeling and optimization of batch cooling crystallization, http://books. google.co.in/books?id=3VMYmwEACAAJ

Worlitschek J and Mazzotti M 2004 Model-based optimization of particle size distribution in batch-cooling crystallization of paracetamol. Cryst. Growth. Des. 4: 891-903

Yang J, McCoy B J and Madras G 2005 Distribution kinetics of polymer crystallization and Avrami equation. J. Chem. Phys. 122(6): 064901C1-10

Yang G, Kutanen M, Sha Z and Kallas J 2006 Determination of operating conditions for controlled batch cooling crystallization. Chem. Eng. Technol. 29(2): 200-205

Zauner R and Jones A G 2002 On the influence of mixing on crystal precipitation processes-application of the segregated feed model. Chem. Eng. Sci. 57: 821-831

Zhou G X, Fujiwara M, Woo X Y, Rusli E, Tung H H, Starbuck C, Davidson O, Ge Z and Braatz R D 2006 Direct design of pharmaceutical anti-solvent crystallization through concentration control. Cryst. Growth. Des. 6(4): 892-898 